\title{
“受阻”路易斯酸碱对催化氢化反应的研究进展
}

\author{
王辉郑 亿潘振涛傅鸿樑凌飞钟为慧* \\ (浙江工业大学药学院 长三角绿色制药协同创新中心 杭州 310014)
}

\begin{abstract}
摘要 “受阻” 路易斯酸碱对(FLPs)催化氢化反应是当前催化加氢领域的研究热点之一. 该类反应具有环境友好、无 重金属残留等特点，具有潜在的工业化应用前景. 根据底物类别，对近几年” 受阻” 路易斯酸碱对在催化氢化领域的研 究进展进行简要评述, 并对 FLPs 不对称催化氢化进行简单介绍.
\end{abstract}

关键词 “受阻”路易斯酸碱对；催化氢化; 不对称催化; 进展

\section{Progress of Frustrated Lewis Pairs in Catalytic Hydrogenation}

\author{
Wang, Hui $\quad$ Zheng, Yi $\quad$ Pan, Zhentao Fu, Hongliang $\quad$ Ling, Fei $\quad$ Zhong, Weihui* \\ (Collaborative Innovation Center of Yangtze River Delta Region Green Pharmaceuticals, College of Pharmaceutical Science, \\ Zhejiang University of Technology, Hangzhou 310014)
}

\begin{abstract}
Frustrated Lewis pairs (FLPs) catalyzed hydrogenation reaction is one of the hotspots in the current hydrogenation field. This kind of reaction has the advantages of environment friendly, no metal residue, etc., and has a potential prospect for industrial application. According to the category of the substrate, a brief review of the recent progress in the field of the FLPs-catalyzed hydrogenation as well as the asymmetric hydrogenation is depicted.

Keywords frustrated Lewis pairs; catalytic hydrogenation; asymmetric catalysis; progress
\end{abstract}

催化氢化反应是指在催化剂作用下氢加成到有机 化合物的不饱和基团上的反应. 在过去的几十年中, 其 催化剂往往采用以钓 $(\mathrm{Ru})$ 、铑 $(\mathrm{Rh})$ 、钯 $(\mathrm{Pd})$ 、铂 $(\mathrm{Pt})$ 、铱 (Ir)、锇 $(\mathrm{Os})$ 等为核心的过渡金属. 尽管过渡金属催化氢 化反应硕果累累, 但逐渐暴露出价格昂贵, 重金属残留 等问题. 尤其是医药行业对食品药品中重金属标准的提 高, 在一定程度上限制了贵金属催化剂在原料药及中间 体合成中的应用.

作为金属催化氢化的补充, 非金属参与的催化氢化 反应由于缺少合适的催化剂而报道相对较少. 直到 2006 年, Stephen 等 ${ }^{[1]}$ 在研究路易斯酸 $\mathrm{B}\left(\mathrm{C}_{6} \mathrm{~F}_{5}\right)_{3}(\mathbf{1})$ 与路易 斯碱 2 反应时发现, 它们没有形成稳定的路易斯酸碱加 合物，而是发生亲核取代反应生成两性离子化合物 $\mathbf{3}$, 将 3 与二甲基氯硅烷反应得到空气以及湿度稳定的两性 离子化合物 4. 化合物 4 加热到 $150{ }^{\circ} \mathrm{C}$ 时可以释放出 $\mathrm{H}_{2}$ 生成化合物 $\mathbf{5}$, 而在 $25{ }^{\circ} \mathrm{C}$ 化合物 $\mathbf{5}$ 可以吸收 $\mathrm{H}_{2}$ 重新生 成 4 (Scheme 1), 过程伴随着明显的颜色变化(图 1). 随 后，Stephan 等提出 “受阻” 路易斯酸碱对(Frustrated
Lewis Pairs, FLPs)的概念，即当大位阻的路易斯酸与路 易斯碱混合，由于空间位阻大而不能形成经典加合物， 从而表现出一些特殊的反应活性.

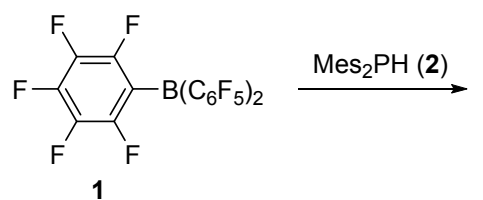<smiles></smiles>

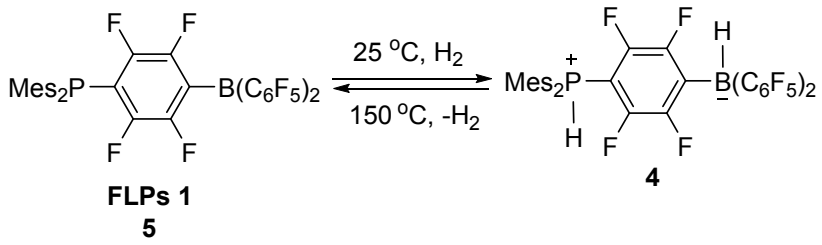

图式 1 “受阻” 路易斯酸碱对可逆活化氢气的发现

Scheme 1 Discovery of reversible $\mathrm{H}_{2}$ activation by FLPs

这个简单的概念一经提出，迅速成为当下研究的热 点, 受到越来越多的关注与重视, 也为无机化学领域开

\footnotetext{
* Corresponding author. E-mail: weihuizhong@zjut.edu.cn

Received July 30, 2016; revised September 19, 2016; published online November 3, 2016.

Project supported by the National Natural Science Foundation of China (Nos. 21276238, 21676253).

国家自然科学基金(Nos. 21276238, 21676253)资助项目.
} 


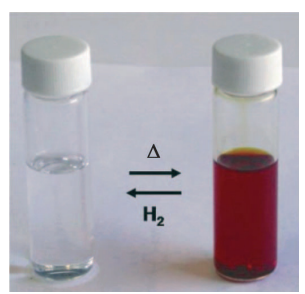

图 1 FLPs 吸收及释放 $\mathrm{H}_{2}$ 的颜色变化

Figure 1 Color changed by FLPs absorb and release $\mathrm{H}_{2}$

辟了新的研究方向. 这种可逆活化 $\mathrm{H}_{2}$ 方法的发现, 使 FLP 在催化氢化领域得到了前所未有的应用, 打开了 FLPs 催化剂在 “非金属” 催化氢化领域的大门.

随后, FLPs 活化 $\mathrm{H}_{2}$ 的研究层出不穷. Erker 等 ${ }^{[2]}$ 报道 了乙基桥连的 $\mathrm{P} / \mathrm{B}$ 体系分子内 FLPs 2, 室温下在戊烷溶 剂中与 $\mathrm{H}_{2}$ 结合形成稳定的两性离子, 收率为 $60 \%$ (Eq. 1). Repo 等 ${ }^{[3]}$ 报道了 $\mathrm{N} / \mathrm{B}$ 体系分子间 FLPs 3 , 与 $\mathrm{H}_{2}$ 反应 得到产物收率为 $95 \%$ (Eq. 2). Tamm 等 ${ }^{[4]}$ 报道了 $\mathrm{C} / \mathrm{B}$ 体 系分子间 FLPs 4, 通过对 $\mathrm{H}_{2}$ 异裂得到两性离子产物, 收率为 $82 \%$ (Eq. 3). 除此之外, 通过改变 Lewis 碱性中 心 $\mathrm{N} / \mathrm{P} / \mathrm{C}$ 上取代基以及硼试剂的酸碱性, 得到很多分子 内或者分子间 FLPs 体系, 实现 $\mathrm{H}_{2}$ 的活化 ${ }^{[5 \sim 11]}$.

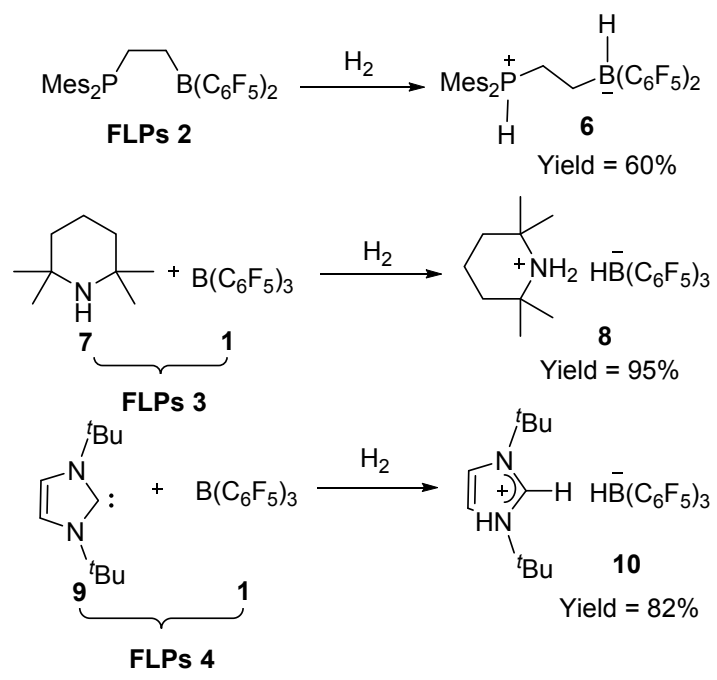

经过 10 年的发展, FLPs 在 $\mathrm{H}_{2}$ 活化领域已经取得了 许多突破性进展 ${ }^{[12,13]}$. 本文从底物官能团类型出发, 主 要综述了近几年 FLPs 催化还原亚胺、烯烃、炔烃、羰 基化合物、芳香族化合物等底物的研究与进展, 并对 FLPs 在不对称催化氢化中的应用进行了介绍, 以及对 该理论未来的发展进行展望.

\section{FLPs 催化还原亚胺}

亚胺的还原是制备饱和胺的最佳方法之一, 并已在 制药和精细化工产业发挥巨大作用. 由于过渡金属催化 容易导致金属残留问题, 以 FLP 为代表的非金属催化还
原亚胺的方法逐渐受到重视.

2007 年, Stephen 等 ${ }^{[14]}$ 报道在甲苯溶剂中, 一定温度 $\left(80 \sim 140{ }^{\circ} \mathrm{C}\right)$ 及 $\mathrm{H}_{2}$ 压力下 $(101 \sim 505 \mathrm{kPa})$ 利用催化剂 FLPs 1 实现亚胺的还原，得到 57\% 99\%的收率(Eq. 4). 若底物为缺电子亚胺, 反应需要更高的压力及温度, 反 应时间也更久. 该反应为 FLPs 催化还原亚胺的首次报 道, 拓展了 FLPs 的研究领域, 也预示着由 $\mathrm{NaBH}_{4}$ 或 $\mathrm{LiAlH}_{4}$ 等催化的还原反应中催化剂的用量可能由化学 计量转变成催化量, 从而大大减少三废的排放.

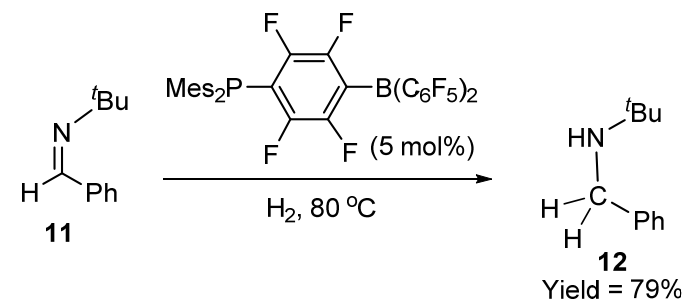

经过研究发现, 亚胺的还原反应是由亚胺的质子化 引发的, 可能机理: 首先 FLPs 1 吸收 $\mathrm{H}_{2}$ 生成两性离子 化合物 14 , 接着 14 上质子转移到底物亚胺上形成中间 体 13 , 最后与氢负离子再转移到底物上得到还原产物 12, 并释放出催化剂(Scheme 2).

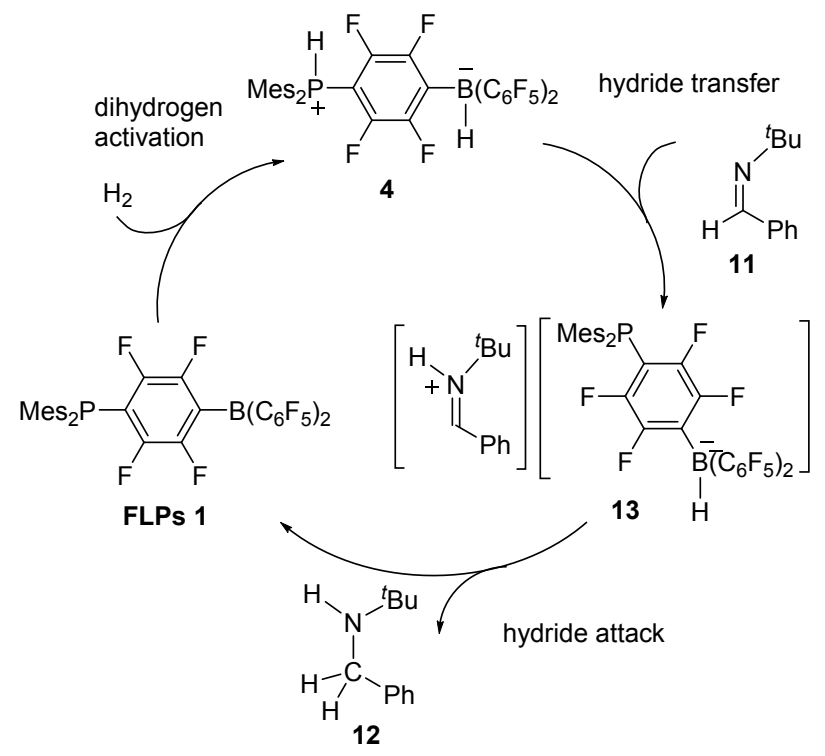

图式 2 FLPs 1 催化还原亚胺的可能机理

Scheme 2 Possible mechanism of imine catalytic reduction by FLPs 1

2008 年, Stephen 等 ${ }^{[15]}$ 研究发现商品化的 Lewis 酸 $\mathrm{B}\left(\mathrm{C}_{6} \mathrm{~F}_{5}\right)_{3}$ 是一种高效的催化剂, 由于五氟苯强吸电子效 应，使得 $\mathrm{B}\left(\mathrm{C}_{6} \mathrm{~F}_{5}\right)_{3}$ 具有一个很强的酸性中心，可以与亚 胺原位生成 FLP, 在氢气条件下实现亚胺的还原. 他们 以甲苯作溶剂, 利用 1 在 $120{ }^{\circ} \mathrm{C}$ 及 $\mathrm{H}_{2}(505 \mathrm{kPa})$ 氛围中 反应 $41 \mathrm{~h}$ 实现亚胺 14 的还原，产物收率为 $94 \%$ (Eq. 5). 
如果在相同反应条件下, 加入额外 $5 \mathrm{~mol} \%$ 的 $\mathrm{P}\left(\mathrm{C}_{6} \mathrm{H}_{2}\right.$ $\left.\mathrm{Me}_{3}\right)_{3}$, 反应时间缩短至 $8 \mathrm{~h}$, 收率稍有提高(Eq. 6). 可能 原因是 $\mathrm{P} / \mathrm{B}$ 体系 FLPs 相对于 N/B 体系还原亚胺的活性 更高, 因此加入 $\mathrm{P}\left(\mathrm{C}_{6} \mathrm{H}_{2} \mathrm{Me}_{3}\right)_{3}$ 表现出促进反应的作用.

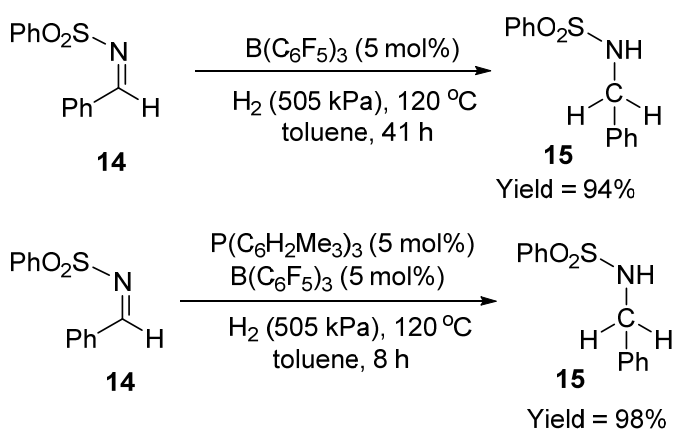

选择性还原肜亚胺的碳氮双键一直比较困难, 而采 用均相的过渡金属催化肟亚胺类衍生物往往直接得到 伯胺. 2014 年, Oestreich 等 ${ }^{[16]}$ 报道了利用 $\mathrm{B}\left(\mathrm{C}_{6} \mathrm{~F}_{5}\right)_{3}$ 选择 性还原肟亚胺而不断裂 $\mathrm{N}-\mathrm{O}$ 键. 以甲苯作溶剂, 10.1 $\mathrm{MPa} \mathrm{H}_{2}$ 压力下, $5 \mathrm{~mol} \%$ 的 $\mathbf{1}$, 实现肜亚胺的还原, 收率 为 $66 \% \sim 99 \%$ (Eq. 7).

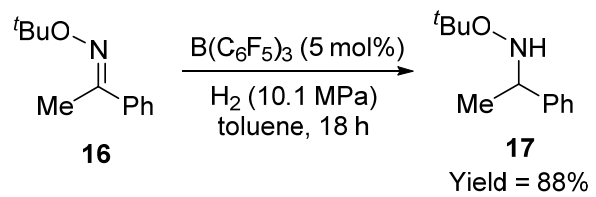

2016 年, 杜海峰等 ${ }^{[17]}$ 报道同样利用 $\mathrm{B}\left(\mathrm{C}_{6} \mathrm{~F}_{5}\right)_{3}$ 在 $\mathrm{H}_{2}$ $(202 \mathrm{kPa})$ 下直接还原 3 位取代的 1,4-苯并恶嗪 18, 收率 为 93\% 99\% (Eq. 8). 该反应的突出优点是催化剂用量 相对较少.

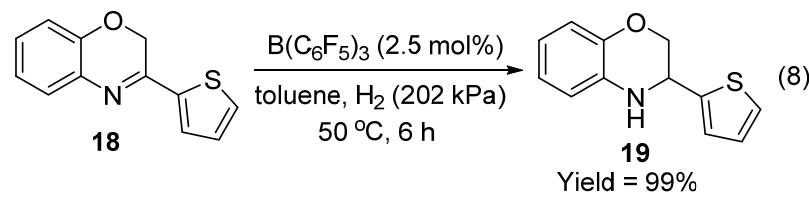

亚胺的还原往往采用 $\mathrm{H}_{2}$ 作为氢源, 存在一定的危 险性. 2011 年, Stephan 等 ${ }^{[18]}$ 报道以二异丙胺为氢源, 1 为 催化剂, 实现亚胺的催化氢化, 收率为 37\% 98\% (Eq. 9). 随后, Oestreich 等 ${ }^{[19]}$ 以 1,5-二甲基-1,4-环已二烯为氢 源, $125{ }^{\circ} \mathrm{C}$ 甲苯作溶剂, 同样在 $\mathrm{B}\left(\mathrm{C}_{6} \mathrm{~F}_{5}\right)_{3}$ 的催化下还原 亚胺, 收率最高可达 $99 \%$ (Eq. 10). 该类反应无需高压, 避免了 $\mathrm{H}_{2}$ 使用存在的高压危险, 为寻找开发新型氢源 提供宝贵的参考.

通过对 $\mathrm{B}\left(\mathrm{C}_{6} \mathrm{~F}_{5}\right)_{3}$ 结构进行适当改造, 可以得到各种 新型 Lewis 酸. 2009 年, Berke 等 ${ }^{[20]}$ 报道利用 1,8 -二五氟 苯基硼基菜在 $d$-甲苯溶剂中 $120{ }^{\circ} \mathrm{C}$ 下, $\mathrm{H}_{2}(1.52 \mathrm{MPa})$ 氛 围中实现亚胺的催化还原, 产物收率最高可达 99\% (Eq.

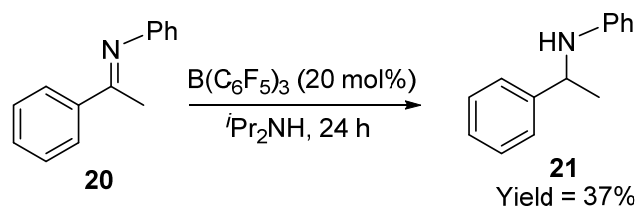<smiles>C/C(=N\F)c1ccccc1</smiles>

20

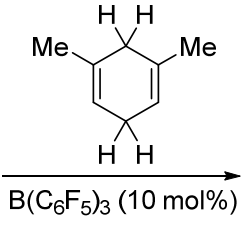

$18 \mathrm{~h}$<smiles>CC(Nc1ccccc1)c1ccccc1</smiles>

11). 需要指出的是, 该反应的限速步在 $\mathrm{H}_{2}$ 的活化, 尽 管该 Lewis 酸具有两个酸性 B 中心，但两个 B 中心共同 活化 $\mathrm{H}_{2}$ 的能垒要比单一 $\mathrm{B}$ 中心的高. 因此, 该 Lewis 酸 可能由一个 $\mathrm{B}$ 中心来活化 $\mathrm{H}_{2}$, 而另一个 $\mathrm{B}$ 中心则表现 出一定的吸电子效应.

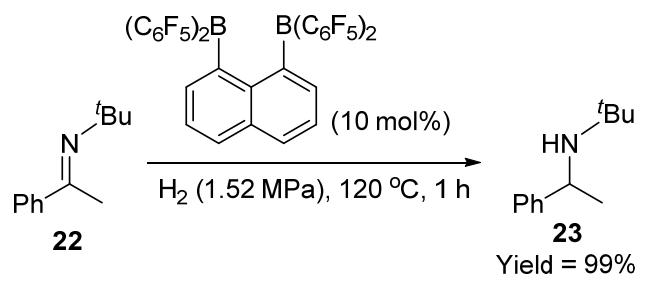

2014 年, Ashley 等 ${ }^{[21]}$ 报道利用 $\mathbf{1}$ 在甲苯中 $80{ }^{\circ} \mathrm{C}$ 还 原亚胺, 当 $\mathrm{H}_{2}$ 压力从 $1.0 \mathrm{MPa}$ 提高到 $3.0 \mathrm{MPa}$ 时, 产物 收率从 7\%提高到了 $99 \%$, 表明压力对反应有很大影响. 随后他们设计合成了 $\mathrm{B}\left(\mathrm{C}_{6} \mathrm{Cl}_{5}\right)\left(\mathrm{C}_{6} \mathrm{~F}_{5}\right)_{2}(\mathbf{2 4}), \mathrm{B}\left(\mathrm{C}_{6} \mathrm{Cl}_{5}\right)_{2}$ $\left(\mathrm{C}_{6} \mathrm{~F}_{5}\right)(25)$ (图 2), 与 1 分别在 $\left[\mathrm{D}_{8}\right] \mathrm{THF}$ 中还原亚胺, 其中 24 的催化收率最高为 $99 \%$. 通过研究发现, 产生不同催 化效果的最主要原因在于三种 Lewis 酸的酸性大小不 同. Lewis 酸 $\mathbf{2 4}$ 的 B 中心酸性介于 1 和 $\mathbf{2 5}$ 之间，可能正 好由于这种适中程度的酸性使得 24 表现出较好的催化 效果.

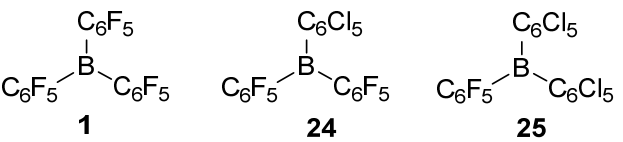

图 2 Lewis 酸 1、24 和 25

Figure 2 Lewis acid 1, 24 and $\mathbf{2 5}$

除了 $\mathrm{B}\left(\mathrm{C}_{6} \mathrm{~F}_{5}\right)_{3}$ 直接还原亚胺之外, 还有很多 FLPs 催化的报道. 2008 年, Erker 等 ${ }^{[22]}$ 报道 FLPs 2 对亚胺进 行还原，在室温及 $253 \mathrm{kPa}$ 下得到 70\%的收率(Eq. 12). 该反应之所以能在温和的条件下进行，得益于 FLPs 2 空间位阻和电子因素之间微妙的平衡关系. 2014 年我们 研究小组 ${ }^{[23]}$ 以环蕃为骨架设计的 FLPs 5 在温和条件下 还原亚胺得到 93\%的收率(Eq. 13); 而且考察了催化剂 的回收套用问题，成功实现三次回收套用且不影响反应 
收率. 从结构可以看出, FLPs 5 与 FLPs 2 的酸碱性中心 $\mathrm{B} / \mathrm{P}$ 是通过两个碳原子相连, 都具有一个适宜空间位阻 的 “活性口袋”, 从而表现出较高的催化活性.
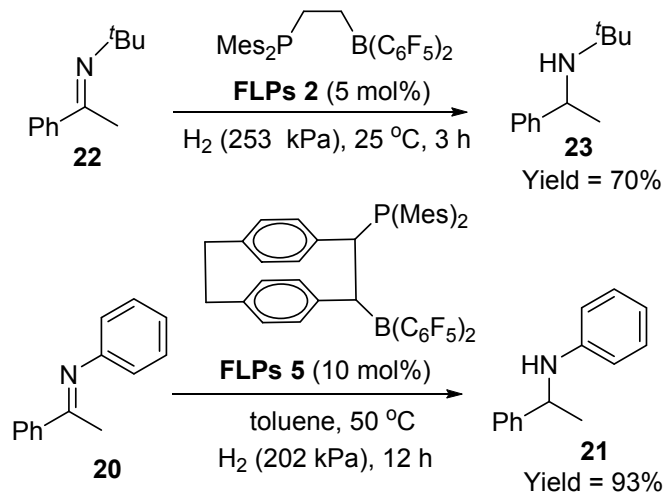

2011 年, 为了考察空间位阻以及路易斯碱性对 FLPs 催化剂活性的影响, Repo 等 ${ }^{[24]}$ 设计了 FLPs 6 8(图 3), 用于亚胺的还原, 其中 FLPs 8 表现出较佳的催 化活性. 可能是由于 FLPs 8 的胺部分具有适宜的空间 位阻和碱性, 使活性中心 N/B 的受阻程度适中, 从而表 现出较大的活性.

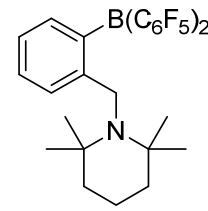

FLPs 6
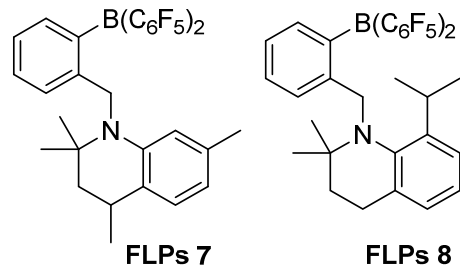

FLPs 8
图 3 N/B 催化剂 FLPs 6 FLPs 8

Figure 3 N/B catalysts FLPs $6 \sim$ FLPs 8

2015 年, Stephan 等 ${ }^{[25]}$ 报道了 C/B 体系催化剂 FLPs 9 催化亚胺、烯胺的反应, 所得产物具有较高的收率(Eq. 14). 该反应催化剂对官能团具有一定耐受性, 底物适 用范围相对较广。

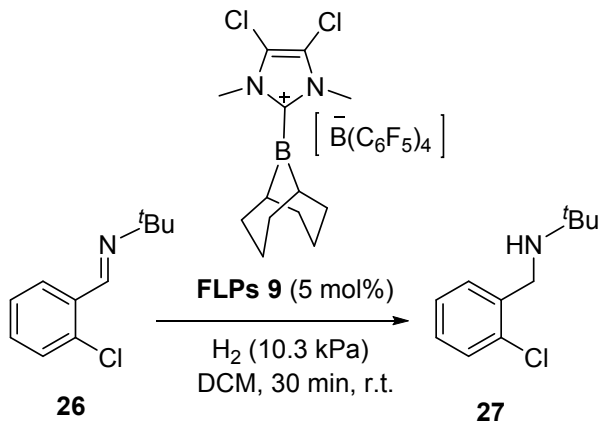

在 FLPs 催化剂的组成中, 路易斯酸由于强吸电子 与大位阻的要求, 其种类受到一定限制, 主要以多卤代 芳基硼为主. 最近, Krempner 等 ${ }^{[26]}$ 基于拓展路易斯酸种 类的考虑, 发现提高路易斯碱的碱性, 可以适当降低路
易斯酸的酸性. 他们利用商业化的超级有机碱 $\mathbf{2 8}$, 与酸 性较弱的路易斯酸 29 结合形成 FLPs 10, 将它与亚胺共 同暴露在 $\mathrm{H}_{2}$ 氛围中，发现亚胺被还原成饱和胺，收率高 达 99\% (Eq. 15). 该反应拓展了路易斯酸碱的种类，在 温和条件下实现亚胺的高效还原.

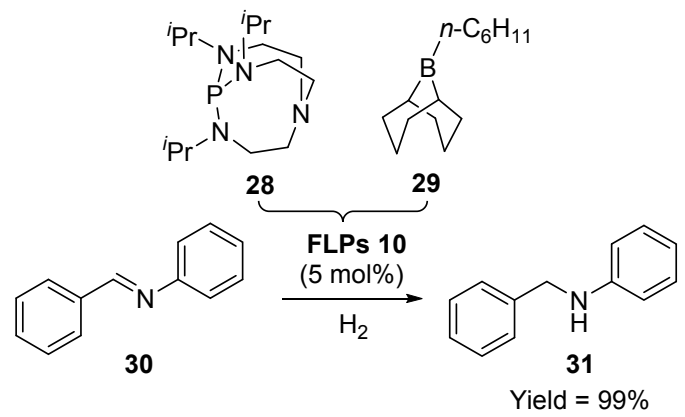

通过上述报道可以发现, FLPs 催化剂主要有 P/B 体 系、 $N / B$ 体系、 $C / B$ 体系等. 一般来说催化剂活性 $P / B>N / B>C / B$. 通过调控催化剂活性中心的空间位阻 和酸碱性, 可以增大 FLPs 催化剂的活性.

\section{FLPs 催化还原烯烃及炔烃}

2008 年, Erker 等 ${ }^{[22]}$ 报道在室温及 $\mathrm{H}_{2}(253 \mathrm{kPa})$ 条件 下，利用 FLPs 2 实现 32 的还原，产物收率为 70\% 99\% (Eq. 16). 2011 年，在 FLPs 2 催化剂基础之上, Erker 等 ${ }^{[27]}$ 将烯胺 32 与 Piers 硼 ${ }^{[28]} 34$ 结合形成分子内 N/B FLPs 11, 在温和条件下异裂 $\mathrm{H}_{2}$ 实现自身底物的还原 (Scheme 5). 与 FLPs 2 相比, FLPs 11 的催化效果相对较 差, 可能是由于 FLPs 11 胺部位的碱性较弱和空间位阻 较小所致.
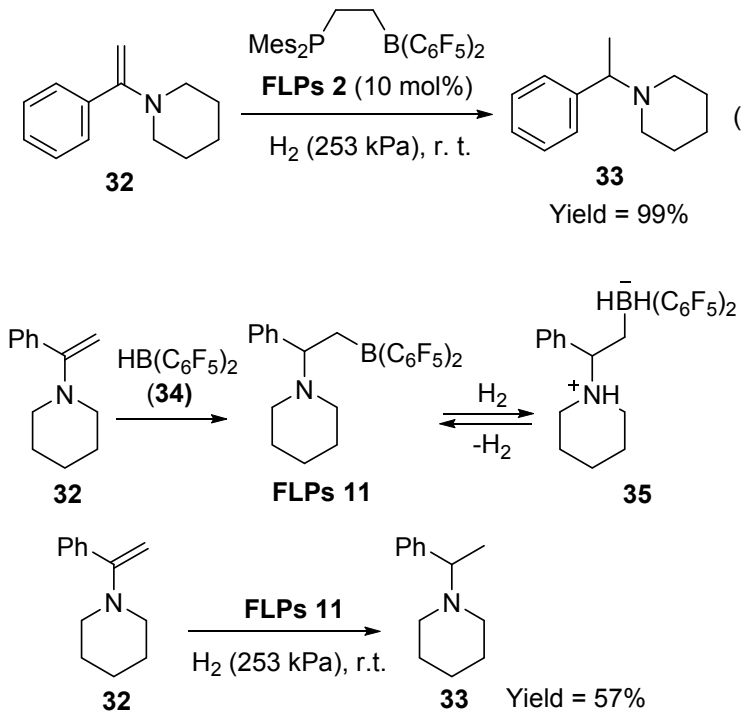

图式 3 FLPs 11 的合成及催化还原烯胺

Scheme 3 Synthesis of FLPs 11 and catalytic reduction of enamine 
2008 年, Erker 等 ${ }^{[29]}$ 利用 1,8 -二二苯基膦荎(36)与 $\mathbf{1}$ 混合, 催化还原烯醇硅醚, 收率为 85\% 93\% (Eq. 17). 该反应较早地报道了 FLPs 对双键的催化还原, 为 FLPs 还原双键的研究奠定了基础. 2012 年, Paradies 等 ${ }^{[30]}$ 报道 了碱性稍弱的 $\left(\mathrm{C}_{6} \mathrm{~F}_{5}\right) \mathrm{Ph}_{2} \mathrm{P}(\mathbf{3 7})$ 与 $\left(\mathrm{C}_{6} \mathrm{~F}_{5}\right) \mathrm{Ph}_{2} \mathrm{P}(\mathbf{3 8})$, 与 1 混 合用于还原烯烃. 不同的是, 37/1 的催化收率可达 99\% 而 38/1 不能催化反应. 从 Lewis 碱结构分析, 可能是 $\mathbf{3 8}$ 的取代基吸电子能力太强, 使 38 的碱性降低至不能实 现催化还原的范围(图 4).
36<smiles>Pc1cccc2c(P)cccc12</smiles><smiles>FC(F)(F)P(c1ccccc1)c1ccccc1</smiles>

37<smiles>FC(F)(F)P(c1ccccc1)C(F)(F)F</smiles>

38
图 4 Lewis 碱 $\mathbf{4 2 \sim 4 4}$

Figure 4 Lewis bases $\mathbf{4 2} \sim \mathbf{4 4}$

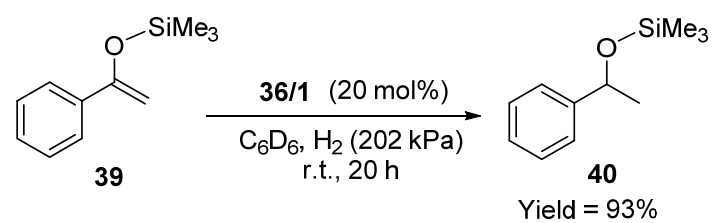

2010 年, Soós 等 ${ }^{[31]}$ 提出了 “排阻设计” (Size exclusion)的概念, 他们认为增加对路易斯酸性中心的屏 蔽作用, 可以改变构成的 FLPs 对 $\mathrm{H}_{2}$ 的活化能力, 可能 会提高 FLPs 的化学选择性. 因此他们设计合成了新型 Lewis 酸 $\mathrm{B}\left(\mathrm{C}_{6} \mathrm{~F}_{5}\right)_{2}(\mathrm{Mes})$, 与 $\mathrm{DABCO}$ 混合催化还原烯烃, 产物收率为 $92 \%$ (Eq. 18).

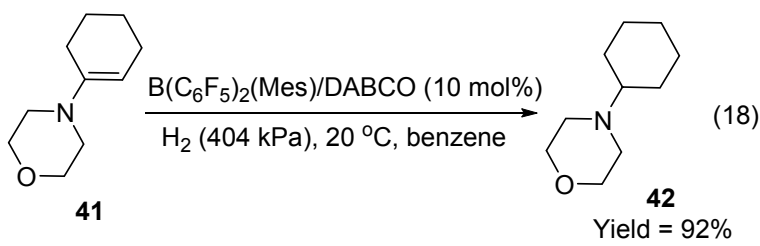

2012 年, Alcarazo 等 ${ }^{[32]}$ 将 1 与 DABCO 混合, $80{ }^{\circ} \mathrm{C}$ 甲苯中选择性还原缺电子烯烃化合物, 产物收率为 64\% 94\% (Eq. 19). 他们报道了系列缺电子烯烃的还 原, 但未见硝基烯烃的还原. 2013 年, Paradies 等 ${ }^{[33]}$ 根据 1 设计合成了酸性稍弱的 $\mathrm{B}\left(\mathrm{C}_{6} \mathrm{H}_{3} \mathrm{~F}_{2}\right)_{3}$, 与 7 组成 FLPs 12 , 在室温下迅速还原硝基烯烃 45, 产物收率可达 $99 \%$ (Eq. 20). 该反应通过调节 Lewis 酸的酸性, 实现对硝基的耐 受性, 预示着在官能团耐受性研究中可以考虑采用调节 Lewis 酸性的方法.

2013 年, Stephan 等 ${ }^{[34]}$ 报道了 1 在乙梄溶剂中实现烯 烃的氢化，收率高达 99\% (Scheme 4)。该反应利用溶剂 作为路易斯碱, 参与 FLPs 催化反应, 大大提高了路易 斯酸的反应活性.
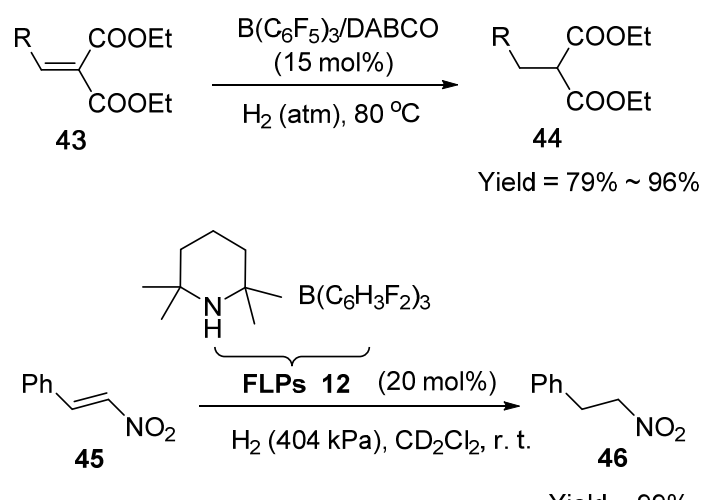

Yield $=99 \%$

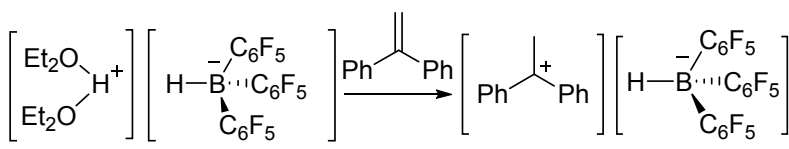

48

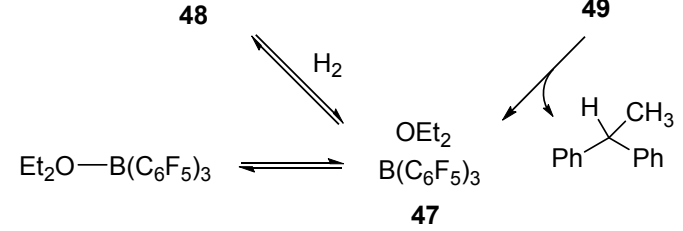

图式 $4 \quad \mathrm{~B}\left(\mathrm{C}_{6} \mathrm{~F}_{5}\right)_{3}$ 与 $\mathrm{Et}_{2} \mathrm{O}$ 组合催化还原烯烃

Scheme 4 Catalytic reduction of olefins by $\mathrm{B}\left(\mathrm{C}_{6} \mathrm{~F}_{5}\right)_{3}$ and $\mathrm{Et}_{2} \mathrm{O}$

2014 年, Erker 等 ${ }^{[35]}$ 基于具有较高催化活性的 FLPs 2, 设计合成了 FLPs 13 和 FLPs 14(图 5). 其中 FLPs 14 活性较高, 可以催化还原烯烃等底物; 而 FLPs 13 的表 现却比较惨淡. 对比发现，可能是 FLPs 2 和 FLPs 14 的 “活性口袋” 大小适中，从而表现出较高的活性.

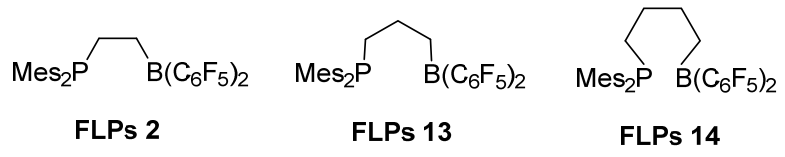

图 5 FLPs 2/13/14 的结构

Figure 5 Structures of FLPs 2/13/14

2013 年, Repo 等 ${ }^{[36]}$ 报道了催化剂 FLPs 15, 实现炔 烃的顺式加氢. 随后他们又拓展了 20 多个炔烃底物, 都 得到了类似的结果, 表明 FLPs 催化剂在部分炔烃的催 化还原中可作为 Lindlar 试剂的替代物. 可能反应机理 首先是 50 异裂 $\mathrm{H}_{2}$ 得到 51, 51 受热后脱去 $\mathrm{C}_{6} \mathrm{~F}_{5} \mathrm{H}$ 结构形 成 FLPs 15, 接着与底物炔烃加成得到 52, 再吸收 $\mathrm{H}_{2}$ 形 成两性离子 53, 随后发生 $\mathrm{H}$ 转移生成顺式加成产物并 游离出催化剂(Scheme 5)

2016 年, Taoufik 等 ${ }^{[37]}$ 报道了有机硅负载的催化剂 FLPs 16 和 FLPs 17 (图 6), 将它们暴露在 $\mathrm{H}_{2}$ 氛围中可以 很好地异裂 $\mathrm{H}_{2}$. 与 FLPs 16 不同的是, FLPs 17 可实现 炔烃的顺式加成, 得到较高的收率. 可能反应机理是: 有机硅负载的 FLPs 17 先与底物加成, 并且硼上的氢转 


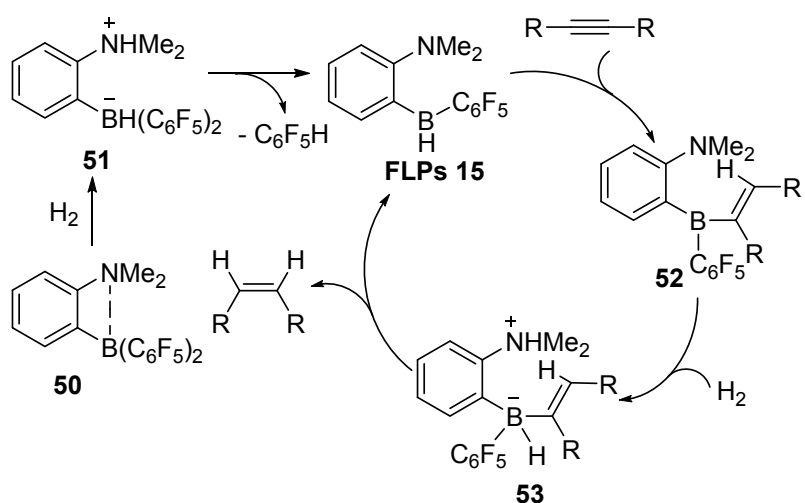

图式 5 FLPs 15 催化炔烃顺式加成可能机理

Scheme 5 Proposed mechanism of the Z-selective reduction of alkynes by FLPs 15

移到不饱和碳上形成 $\mathbf{5 4}$, 接着将 $\mathrm{H}_{2}$ 异裂形成两性离子 55, 由于催化剂较大的空间位阻, 底物上的取代基被挤 到了同侧, 随后路易斯碱上的氢转移到底物上得到顺式 加成产物并且释放出 FLPs 17 (Scheme 6).
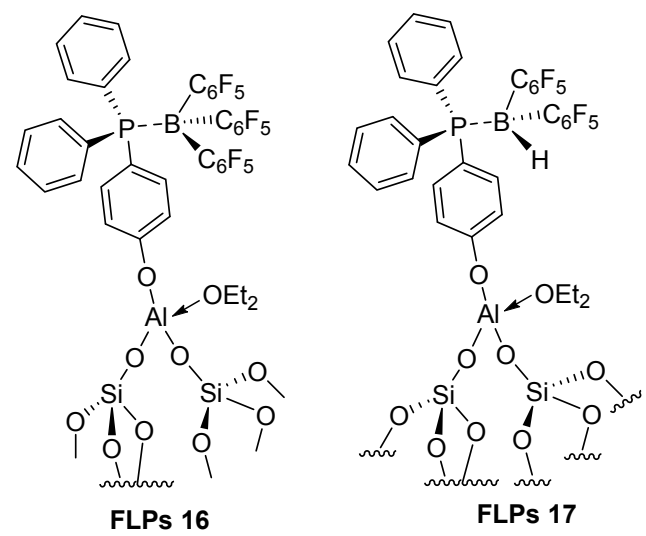

图 6 有机硅负载的催化剂 FLPs 16/17

Figure 6 Organic silicon supported catalyst FLPs 16/17

FLPs 对烯烃及炔烃的还原效果直接与催化剂的结构相 关. 适当的 Lewis 酸碱性和适中的”活性口袋”有利于提 高 FLPs 催化剂的活性; 通过调节多卤代硼的酸性强弱, 有可能提高催化剂的官能团耐受性.

\section{FLPs 催化还原羰基化合物}

由于羰基易与嗍试剂结合，在一定程度上限制了 FLPs 催化羰基化合物的研究. 2012 年, Erker 等 ${ }^{[38]}$ 报道了 催化剂 FLPs 18 (Scheme 7), 与 $\alpha, \beta$-不饱和羰基化合物 共同暴露在 $\mathrm{H}_{2}$ 氛围中, 发现只有双键被还原, 收率为 $74 \%$ ～91\%. 同年, Paradies 等 ${ }^{[39]}$ 报道具有良好刚性和较 大空间位阻的 FLPs 19 与 FLPs 20 (Scheme 7), 在 $\mathrm{HSiPh}_{2} \mathrm{Me}$ 与 $\mathrm{H}_{2}$ 的环境中, 将 $\alpha, \beta$-不饱和羰基化合物的 羰基和双键还原，产物收率为 $58 \% \sim 90 \%$. 其机理是首 先 $\mathrm{HSiPh}_{2} \mathrm{Me}$ 与 1 催化 $\mathbf{5 6}$ 进行 1,4 加成得到 $\mathbf{5 8}$, 接着

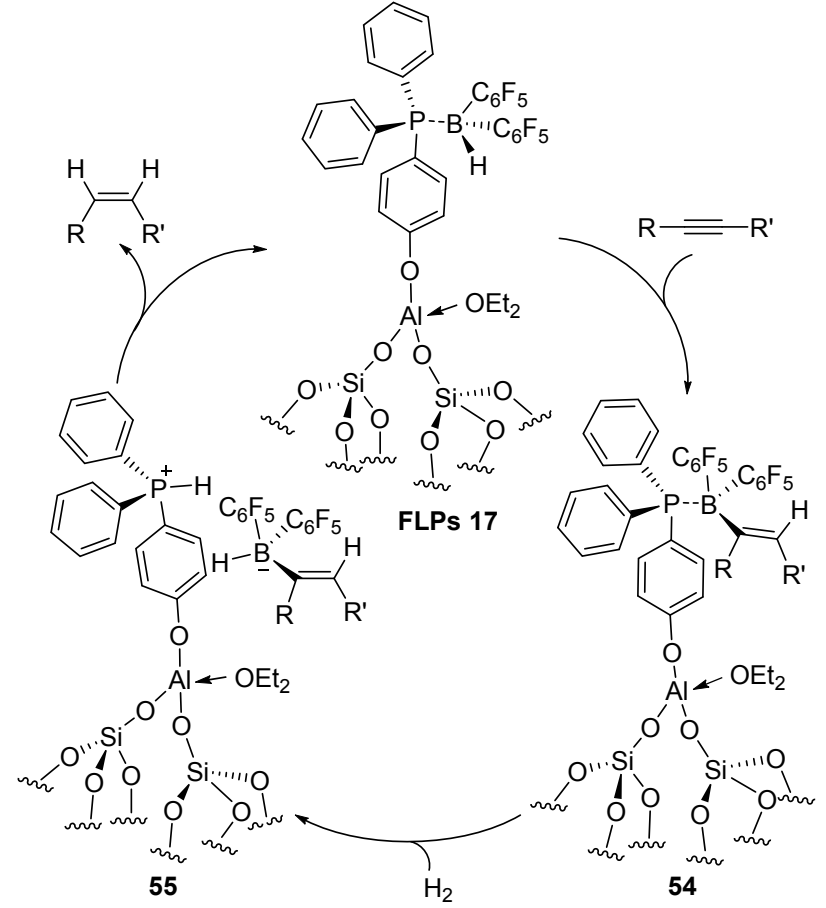

图式 6 FLPs 17 催化炔烃顺式加成可能机理

Scheme 6 Proposed mechanism of the $Z$-selective reduction of alkynes by FLPs 17

FLPs 19/20 在 $\mathrm{H}_{2}$ 氛围中还原 58 得到产物 59.

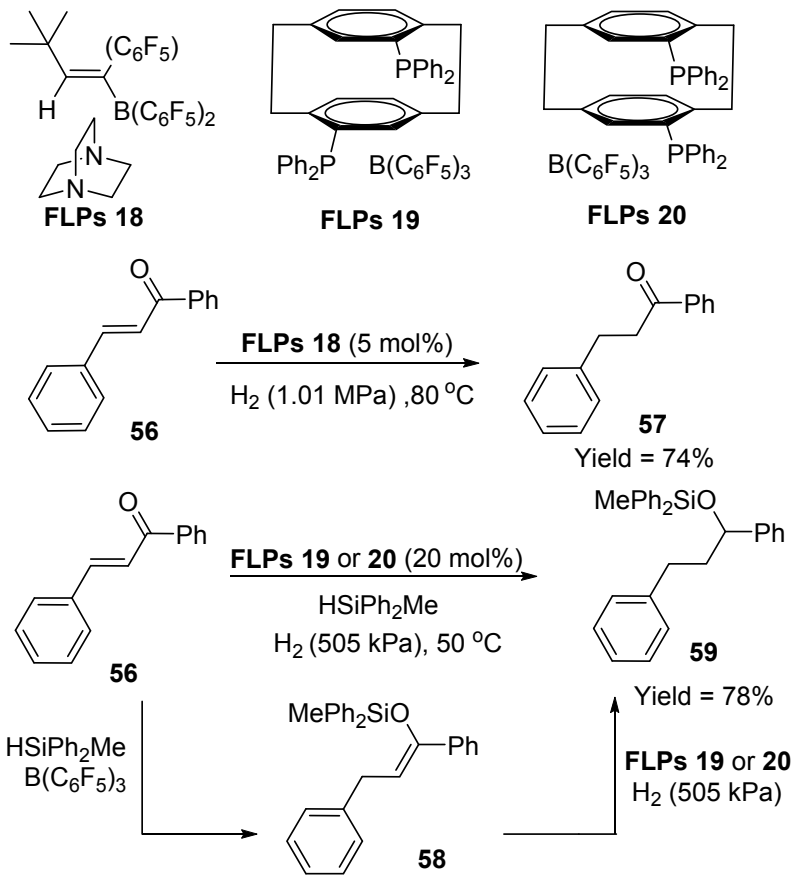

图式 7 新型催化剂 FLPs 18 20

Scheme 7 New catalysts FLPs $\mathbf{1 8} \sim \mathbf{2 0}$

2014 年, Stephan 等 ${ }^{[40]}$ 报道利用 $\mathrm{B}\left(\mathrm{C}_{6} \mathrm{~F}_{5}\right)_{3}$ 在甲苯中 $110{ }^{\circ} \mathrm{C}$ 和 $\mathrm{H}_{2}(404 \mathrm{kPa})$ 条件下, 将酮还原得到硼酯 61 , 收率为 $85 \%$ \% $99 \%$ (Eq. 21). 随后，他们 ${ }^{[41]}$ 将溶剂替换 成乙醚，实现脂肪酮与芳香酮的还原，产物收率为 
53\% 99\% (Eq. 22). 将甲苯替换成醚类溶剂, 只需催化 量的 1 就可将酮直接还原成醇. 从反应机理可以发现, 醚类溶剂可以作为弱的 Lewis 碱参与催化反应. 同样的 结论再一次被 Ashley 等证明, 他们 ${ }^{[42]}$ 以 1,4-二氧六烷作 溶剂, 在较温和的条件下成功地将醛酮化合物还原, 产 物收率为 60\% 99\% (Eq. 23). 然而在 2015 年, Stephan 等 ${ }^{[43]}$ 报道以甲苯作溶剂, 加入环糊精 $(\alpha-C D)$ 或者分子篎

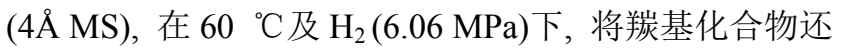
原成醇, 且得到较高的收率(Eq. 24). 该反应中 $\mathrm{B}\left(\mathrm{C}_{6} \mathrm{~F}_{5}\right)_{3}$ 与 $\alpha-C D$ 或 $4 \AA \mathrm{MS}$ 可以组成 FLPs 催化剂, 从而实现催 化反应.

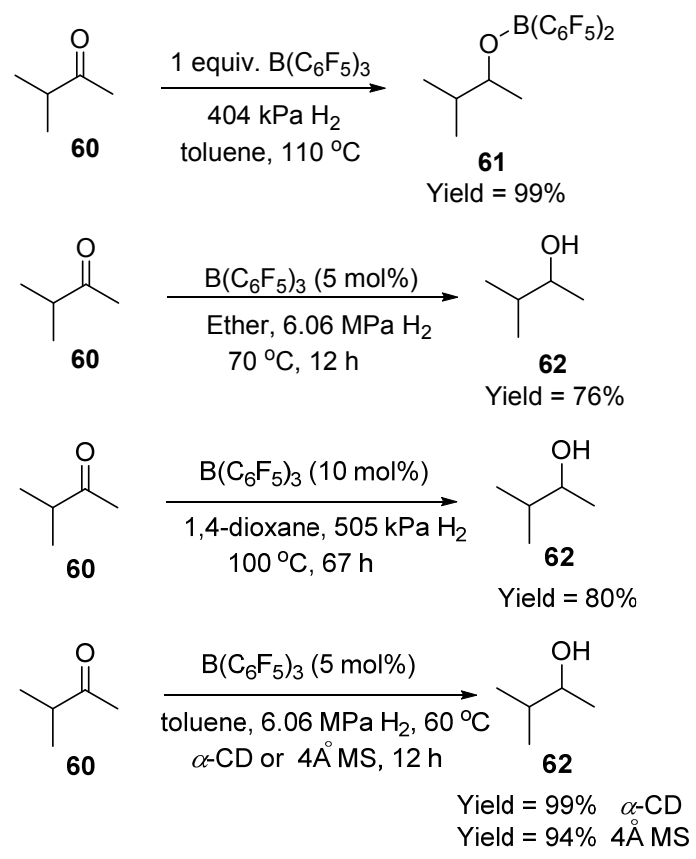

2015 年, Soós 等 ${ }^{[44]}$ 根据 “排阻设计” (Size exclusion) 的概念, 设计合成了新型路易斯酸 63(图 7), 以工业级 THF 作溶剂, 实现醛及酮的催化氢化, 催化剂表现出很 好的耐湿性. 该反应首次报道了 FLPs 在工业级溶剂中 进行催化反应, 预示着适当减弱 Lewis 酸性可能会提高 FLPs 催化剂的耐湿性.

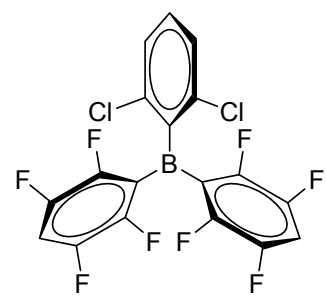

63

图 7 新型路易斯酸 $\left(\mathrm{C}_{6} \mathrm{HF}_{4}\right)_{2} \mathrm{~B}\left(\mathrm{C}_{6} \mathrm{H}_{3} \mathrm{Cl}_{2}\right)$

Figure 7 New Lewis acid $\left(\mathrm{C}_{6} \mathrm{HF}_{4}\right)_{2} \mathrm{~B}\left(\mathrm{C}_{6} \mathrm{H}_{3} \mathrm{Cl}_{2}\right)$

总的来说, FLPs 对羰基化合物还原的报道相对较 少. 在醚类溶剂中利用 $\mathrm{B}\left(\mathrm{C}_{6} \mathrm{~F}_{5}\right)_{3}$ 可以实现羰基化合物的
催化还原; 而减弱多卤代嗍试剂的酸性有可能增强其对 湿度的稳定性.

\section{FLPs 催化还原芳香族化合物}

以往报道的 FLPs 催化剂主要应用于烯烃、炔烃、 亚胺等的还原，鲜有报道对芳香族化合物的还原. 2010 年, Stephan 等 ${ }^{[45]}$ 发现在氢气氛围中, 将 $\mathrm{B}\left(\mathrm{C}_{6} \mathrm{~F}_{5}\right)_{3}$ 和 $\mathrm{N}$ 杂 芳烃化合物接触，可以选择性地将吡啶部分还原生成四 氢喹啉, 收率为 $74 \% \sim 88 \%$ (Eq. 25). 该反应的缺点是 必须在喹啉的 2 号或 8 号位引入取代基，增大底物空间 位阻，而空间位阻较小的喹啉不适用。该反应首次报道 了 $\mathrm{B}\left(\mathrm{C}_{6} \mathrm{~F}_{5}\right)_{3}$ 直接应用于 $\mathrm{N}$ 杂芳烃化合物的催化加氢反 应, 拓展了底物范围, 为 FLPs 的研究开辟了新的方向.

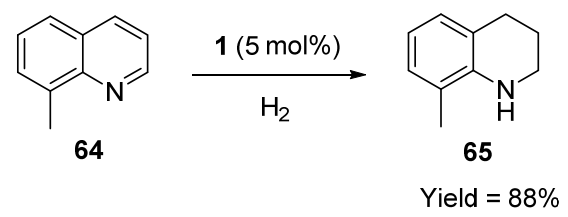

可能的反应机理: 首先发生 $\mathrm{H}_{2}$ 的活化, 底物 64 和 1 与 $\mathrm{H}_{2}$ 作用生成两性离子 $\mathbf{6 6}$, 接着发生 $\mathrm{H}_{2}$ 的 1,4 加成生 成 67 或者 1,2 加成生成 68 并游离出 1 , 接下来 67 或 68 异构化生成 69 , 然后又与 $\mathbf{1}$ 共同活化 $\mathrm{H}_{2}$ 得到两性离子 70, 最后再次发生 $\mathrm{H}_{2}$ 加成得到还原产物 65 并释放出 1 (Scheme 8).

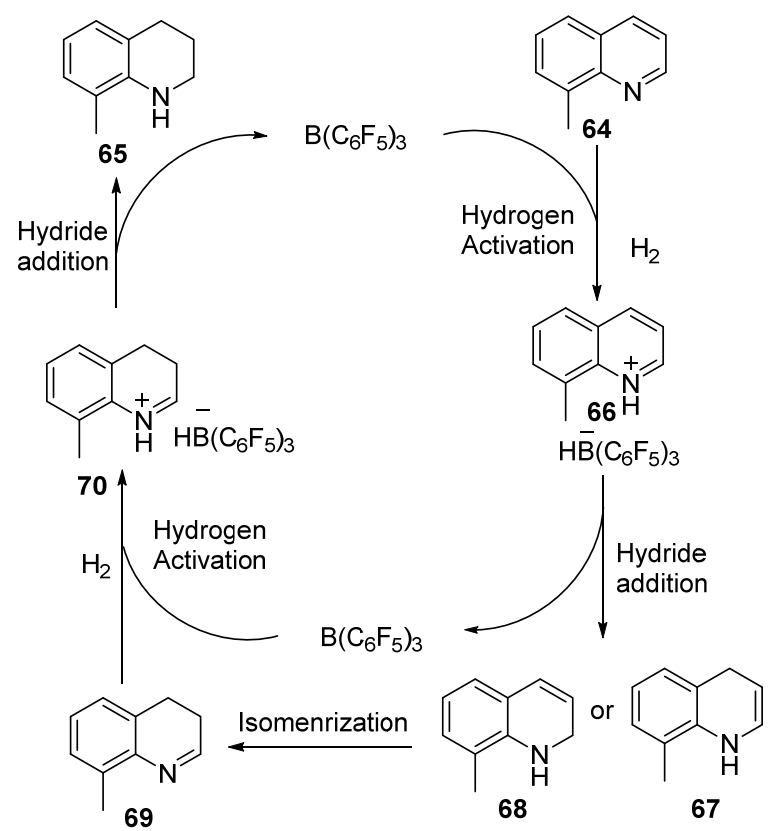

图式 $8 \quad B\left(C_{6} F_{5}\right)_{3}$ 催化还原 $\mathrm{N}$ 杂芳烃化合物可能机理 Scheme 8 Proposed mechanism of catalytic reduction of $\mathrm{N}$ heterocyclic aromatic compounds by $\mathrm{B}\left(\mathrm{C}_{6} \mathrm{~F}_{5}\right)_{3}$

2012 年, Soós 等 ${ }^{[46]}$ 根据 “排阻设计” 的概念报道了 新型 Lewis 酸 $\mathrm{MesB}\left(\mathrm{C}_{6} \mathrm{~F}_{4} \mathrm{H}\right)_{2}(\mathbf{7 1})$, 在 $105{ }^{\circ} \mathrm{C}$ 下实现喹啉 
及衍生物的还原, 得到 79\% 99\%的收率(Eq. 26). 相对 于 1 而言, 71 具有更大的空间位阻和更弱的 Lewis 酸性, 可能正是这些性质使 71 可以很好的还原空间位阻较小 的喹啉及衍生物.

$$
\underbrace{\mathrm{MesB}\left(\mathrm{C}_{6} \mathrm{~F}_{4} \mathrm{H}\right)_{2}}_{\text {Yield }=79 \% \sim 99 \%}
$$

随后, 他们 ${ }^{[47]}$ 将 71 应用到四氢喹啉生物碱枯杷仞 合成中, 将中间体 76 还原得到 77, 收率为 91\% (Scheme 9). 这是 FLPs 催化剂首次用于生物碱的合成, 为 FLPs 催化剂的工业化应用奠定了基础.<smiles>Cc1ccc2ccccc2n1</smiles><smiles>COc1ccc(C=O)cc1OC</smiles>

75<smiles>COc1ccc(/C=C/c2ccc3ccccc3n2)cc1OC</smiles>

76<smiles>COc1ccc(CC[C@@H]2CCc3ccccc3N2C)cc1OC</smiles>

图式 $9 \operatorname{MesB}\left(\mathrm{C}_{6} \mathrm{~F}_{4} \mathrm{H}\right)_{2}$ 应用于枯杷仞合成

Scheme 9 Application of $\operatorname{MesB}\left(\mathrm{C}_{6} \mathrm{~F}_{4} \mathrm{H}\right)_{2}$ in the synthesis of cuspareine

2012 年, Stephan 等 ${ }^{[48]}$ 利用催化剂 FLPs 21, 首次实 现了葸类及其衍生物的还原，收率最高可以达到 $97 \%$, 进一步拓展了 FLPs 催化的底物范围(Eq. 27). 值得注意 的是, 该体系只有含葸结构的多环芳烃可以被还原, 而 荎、萠、菲、醌不能被还原, 可能是由于形成的碳正离 子不够稳定，从而不适用于该催化体系.

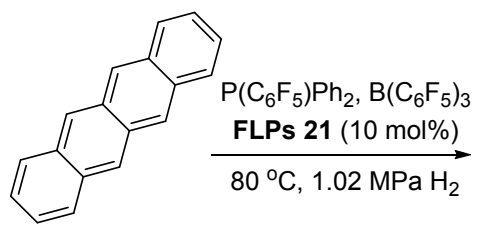

79

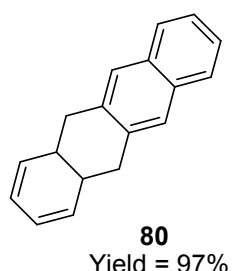

(27)
FLPs 催化多环芳烃反应可能的机理与催化亚胺反 应机理类似, 首先是 FLPs 21 吸收 $\mathrm{H}_{2}$, 形成两性离子化 合物 81, 接着与多环芳烃底物反应, 质子转移到底物生
成碳正离子中间体 83 , 然后 83 接收氢负离子得到还原 产物，并释放出 FLPs 催化剂(Scheme 10).

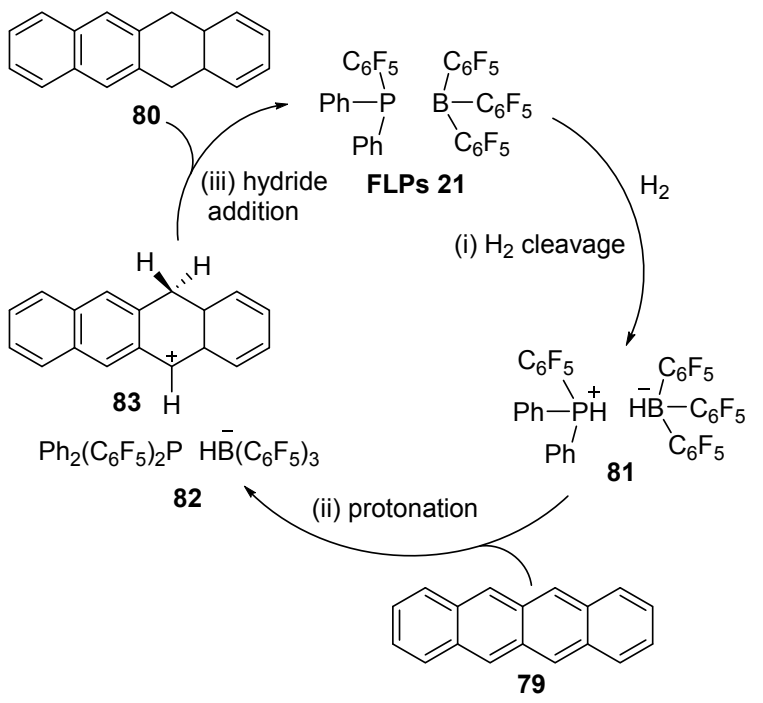

图式 10 FLPs 21 催化还原多环芳烃可能机理

Scheme 10 Proposed mechanism of catalytic reduction polycyclic aromatic hydrocarbons by FLPs 21

2012 年, Stephan 等 ${ }^{[49]}$ 报道将 $\mathbf{1}$ 与芳胺 84 共同暴露 在 $\mathrm{H}_{2}$ 氛围中，室温下发生 $\mathrm{H}_{2}$ 的异裂得到两性离子化合 物 85. 然而, 当温度升至 $110{ }^{\circ} \mathrm{C}$ 时, 发现可将苯环还原 成环己烷，收率良好为 $61 \%$ ～93\% (Scheme 11). 2015 年， Stephan 等 ${ }^{[50]}$ 再次证实了这一现象. 不同的是, 在还原 对甲氧基苯胺时发现(Eq. 28), 对位甲氧基可以被脱去, 而邻位甲氧基则不易被脱去. 从反应机理(Scheme 12) 可以发现，对位甲氧基苯胺还原可以得到中间体 92, 而 邻位取代苯胺则生成中间体 94 , 可能是由于 92 的结构 相对更加稳定，从而表现出对位与邻位的差异.

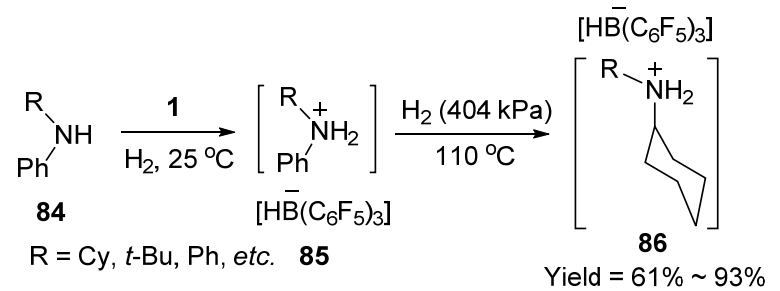

图式 $11 \quad \mathrm{~B}\left(\mathrm{C}_{6} \mathrm{~F}_{5}\right)_{3}$ 催化还原苯环

Scheme $11 \quad B\left(\mathrm{C}_{6} \mathrm{~F}_{5}\right)_{3}$ catalytic reduction of benzene

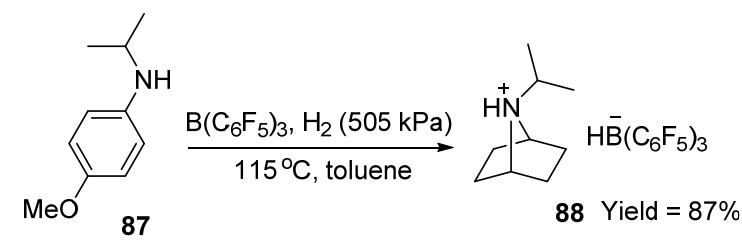

2013 年, Stephan 等 ${ }^{[51]}$ 利用 $\mathbf{1}$ 在甲苯溶剂中, $115{ }^{\circ} \mathrm{C}$ 下与 $\mathrm{H}_{2}$ 反应, 将吡啶衍生物还原得到两性离子化合物, 收率为 $54 \% \sim 92 \%$ (Eq. 29). 随后，杜海峰等 ${ }^{[52]}$ 将烯烃 


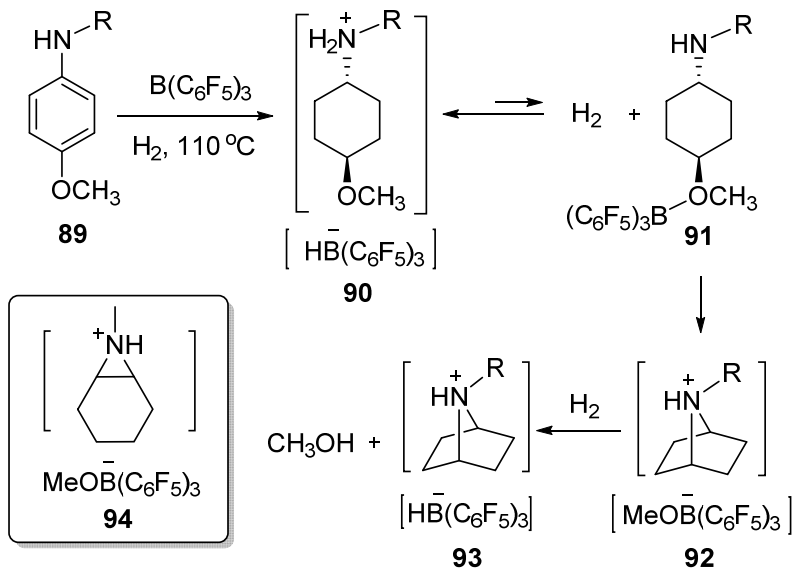

图式 $12 \quad \mathrm{~B}\left(\mathrm{C}_{6} \mathrm{~F}_{5}\right)_{3}$ 催化还原苯环可能机理

Scheme 12 Proposed mechanism of catalytic reduction benzene by $\mathrm{B}\left(\mathrm{C}_{6} \mathrm{~F}_{5}\right)_{3}$

与 Peris 硣原位生成新型 Lewis 酸, 成功实现吡啶类衍生 物的还原, 收率为 80\% 99\% (Eq. 30). 与 Stephan 报道 使用化学计量的 $\mathrm{B}\left(\mathrm{C}_{6} \mathrm{~F}_{5}\right)_{3}$ 不同的是, 杜海峰报道只需催 化量的 1 便可得到较高的收率.

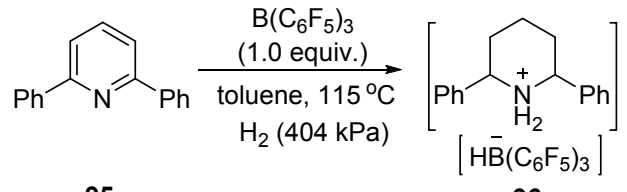

95

$$
96
$$

Yield $=92 \%$

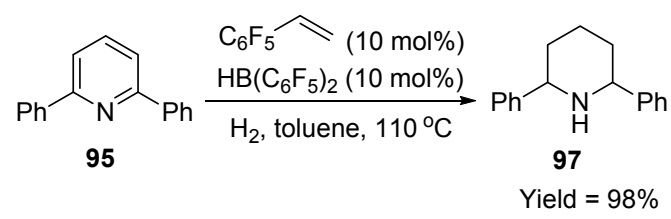

在 FLPs 还原芳香族化合物中, 使用 Lewis 酸直接进 行催化反应的报道较多. 使用化学计量的 $\mathrm{B}\left(\mathrm{C}_{6} \mathrm{~F}_{5}\right)_{3}$ 还原 $\mathrm{N}$ 杂芳烃容易得到两性离子化合物, 而通过调节 $\mathrm{B}\left(\mathrm{C}_{6} \mathrm{~F}_{5}\right)_{3}$ 的酸性和空间位阻得到的新型 Lewis 酸可以在 催化量的条件下直接还原 $\mathrm{N}$ 杂芳烃得到中性还原产物.

\section{FLPs 应用于不对称催化加氢反应}

除了上述加氢反应, FLPs 在不对称催化氢化领域也 表现出独特的活性 ${ }^{[53]} .2008$ 年, Klankermayer 等 ${ }^{[54]}$ 报道 了手性嗍试剂 98, 在甲苯中 $65{ }^{\circ} \mathrm{C}$ 下不对称还原亚胺, 转化率高达 99\%. 尽管产物 $e e$ 值仅 13\% (Eq. 31), 但为 FLPs 在不对称催化氢化领域的研究开辟了道路.

随后, 他们 ${ }^{[5]}$ 以樟脑为骨架制备手性 Lewis 酸 103a 与 $103 \mathrm{~b}$, 由于它们不易分离, 通过直接加入路易斯碱与 $\mathrm{H}_{2}$ 分离得到了 104a 和 104b, 在温和的条件下实现亚胺 的不对称催化还原反应, 产物收率为 $37 \% \sim 99 \%$, ee 值 为 $20 \% \sim 83 \%$ (Scheme 13). 由此表明, 适当骨架的手性

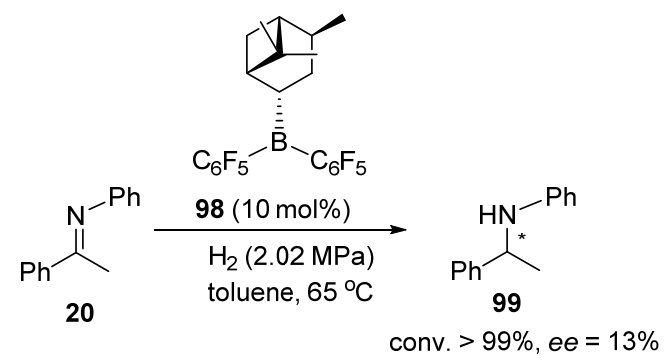

FLPs 催化剂有助于提高催化氢化反应的对映选择性.

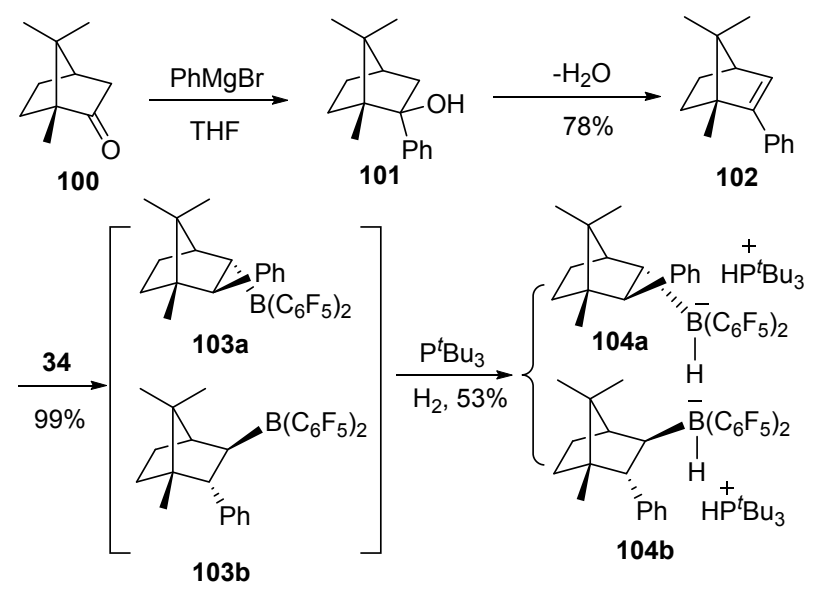

图式 $13103 \mathbf{a} / 103 \mathbf{b}$ 的合成及 $\mathrm{H}_{2}$ 活化

Scheme 13 Synthesis of 103a/103b and activation of $\mathrm{H}_{2}$

除了分子间 $\mathrm{P} / \mathrm{B}$ 手性 FLPs, Klankermayer 等 ${ }^{[56]}$ 又报 道相同骨架的分子内手性 P/B 催化剂 FLPs 22, 将其应 用于亚胺的不对称催化加氢(Eq. 32), 产物收率为 $21 \% \sim 95 \%, e e$ 值为 $70 \% \sim 76 \%$. 该催化剂的突出优点是 对空气稳定, 用量少且可回收使用四次. 2012 年, 他 们 ${ }^{[57]}$ 报道了新型手性催化剂 FLPs 23(图 8), 以 $\mathrm{Me}_{2} \mathrm{PhSiH}$ 为氢源实现亚胺的不对称还原, 得到 $90 \%$ 的 收率和 $84 \%$ 的 $e e$ 值(Eq. 33).
FLPs 22

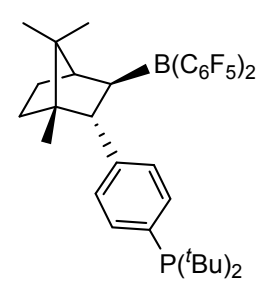

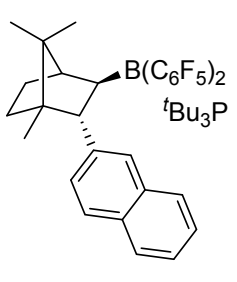

FLPs 23
图 8 手性催化剂 FLPs 22/23

Figure 8 Chiral catalyst FLPs 22/23
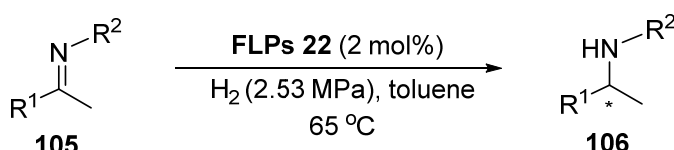

$\mathrm{R}^{1}=\mathrm{Ph}, 4-\mathrm{MeOC}_{6} \mathrm{H}_{4}, 4-\mathrm{ClC}_{6} \mathrm{H}_{4}, 2-$ Naphthyl $\quad$ Yield $=21 \% \sim 95 \%$ $\mathrm{R}^{2}=\mathrm{Ph}, 4-\mathrm{ClC}_{6} \mathrm{H}_{4}, 4-\mathrm{MeOC}_{6} \mathrm{H}_{4}$ ee $=70 \% \sim 76 \%$ 
<smiles>COc1ccc(C(C)Nc2ccccc2)cc1</smiles>

(33)

2011 年, Repo 等 ${ }^{[18 a]}$ 合成了分子内手性 N/B 催化剂 FLPs 24, 首次实现了 $\mathrm{N}$ 杂环化合物 109 的不对称氢化, 拓宽了手性 FLPs 催化剂的底物范围, 反应转化率高达 $100 \%$ (Eq. 34), 但 $e e$ 值仅 37\%, 且反应时间相对较长. 反应 $e e$ 值较低, 可能是由于催化剂手性中心距离较远 或者空间位阻较小导致.

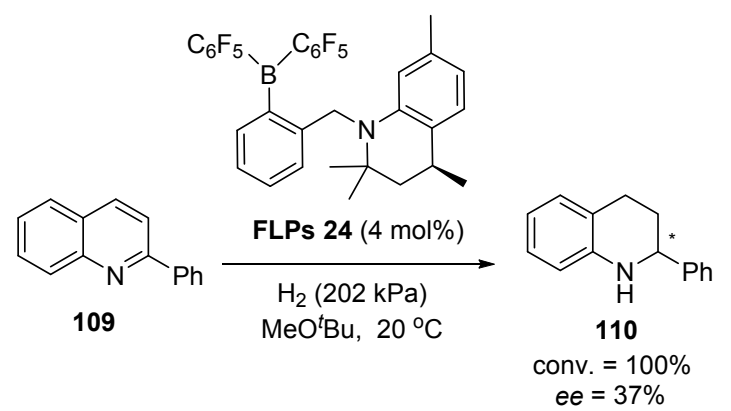

2015 年, Repo 等 ${ }^{[58]}$ 报道合成手性连萗 N/P 体系催化 剂 FLPs 25, 在室温下甲基叔丁基醚中与 $\mathrm{H}_{2}(202 \mathrm{kPa})$ 作 用实现亚胺的不对称还原，产物收率为 34\% 92\%, ee 值为 $32 \% \sim 83 \%$ (Eq. 35). 随后他们又将该催化剂应用 于烯胺的不对称还原, 产物的收率和 ee 值最高可达 99\%. 该反应首次报道了在 FLPs 催化下, 实现烯胺化合 物的不对称还原, 且反应条件温和, 催化剂合成步骤简 单.

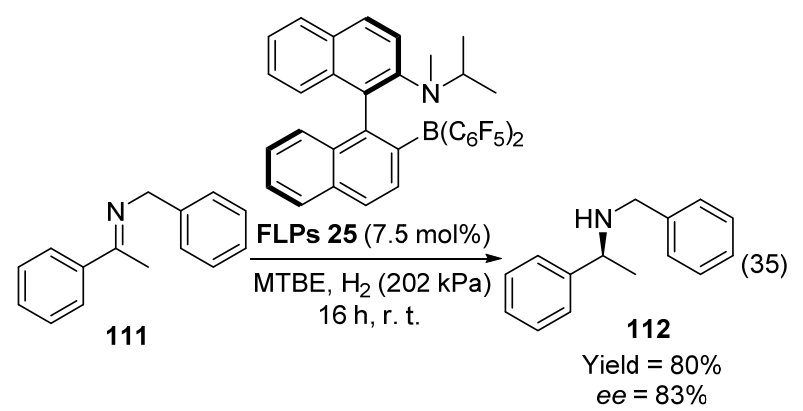

2013 年, Oestreich 等 ${ }^{[59]}$ 报道了手性环硼路易斯酸 113(图 9), 以 $\mathrm{Me}_{2} \mathrm{PhSiH}$ 为氢源实现亚胺的不对称还原, 得到 54\%的收率和 $62 \%$ 的 $e e$ 值(Eq. 36). 2016年, 他们 ${ }^{[60]}$ 根据 113 设计合成了新型 Lewis 酸 114(图 9), 以 $\mathrm{PhSiH}_{3}$ 为氢源, 在室温下实现羰基的手性还原, 得到最高 $87 \%$ 的收率和 $99 \%$ 的 ee 值(Eq. 37). 该反应无需额外加入 Lewis 碱便可实现还原, 为研究开发新型FLPs 催化剂提

供参考.

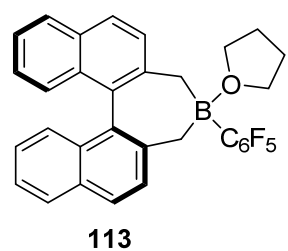<smiles></smiles>

图 9 手性催化剂 FLPs 113/114

Figure 9 Chiral catalyst FLPs 113/114
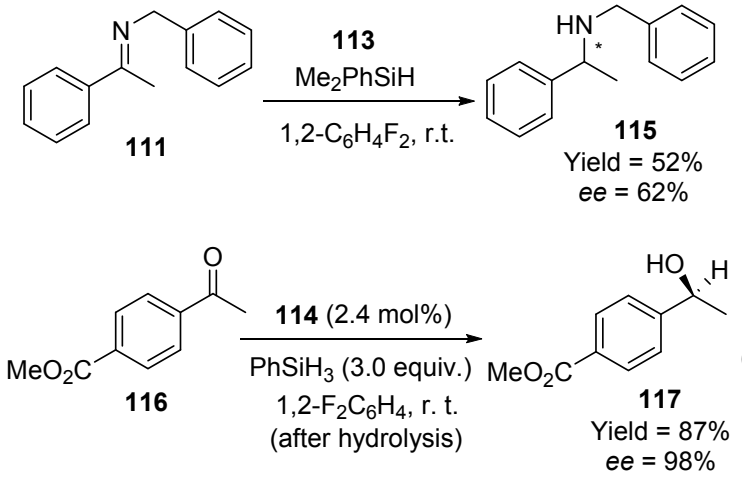

2013 年, 杜海峰等 ${ }^{[61]}$ 合成了手性联芸骨架二烯化 合物，与 34 加成得到 118, 在 $\mathrm{H}_{2}$ 环境中实现亚胺的不对 称还原，产物收率为 63\% 99\%, ee 值为 74\% 89\% (Eq. 38). 该反应催化剂原位生成，无需分离提纯，操作简 便，可直接投入反应，快速而高效地篎选手性烯烃 “配 体”，大大提高反应活性，为 FLPs 催化剂的合成及应用 于不对称催化氢化提供新的思路.

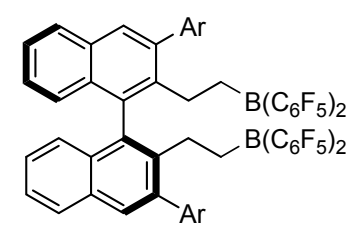

118

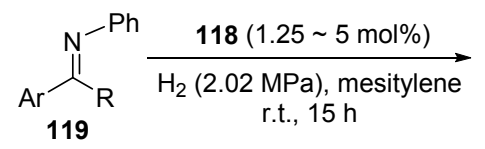

$\mathrm{R}=\mathrm{BnO}, \mathrm{CF}_{3} \mathrm{O}, \mathrm{Me}$, etc

2014 年，杜海峰等 ${ }^{[62]}$ 报道合成了 FLPs 26, 将其应 用于烯醇硅醚的还原，得到 89\% 99\%的收率和 $42 \%$ $98 \%$ 的 $e e$ 值 (Eq. 39). 2015 年, 他们 ${ }^{[63]}$ 又设计了手性催 化剂FLPs 27, 在 $\mathrm{H}_{2}$ 氛围实现不对称催化还原, 产物收 率为 $82 \% \sim 98 \%$, ee 值为 $87 \% \sim 99 \%$ (Eq. 40). 该催化剂 适宜的空间结构和适中的路易斯酸性使其在不对称还 原双键反应中表现出超高的活性(图 10).

2016 年，杜海峰等 ${ }^{[64]}$ 报道了催化剂 FLPs 28, 以 $\mathrm{PhMe}_{2} \mathrm{SiH}$ 为氢源, 对 1,2-二羰基化合物实现不对称还 
(1) FLPs 26 (5 mol\%)<smiles>C=C(O[Na])c1ccc2ccccc2c1</smiles>

$\mathrm{H}_{2}(4.04 \mathrm{MPa})$, toluene

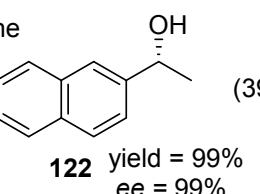

(2) TBAF, toluene

$r, t, 0.5 \mathrm{~h}$

122 ee $=99 \%$<smiles>C=C(OC)c1cccc2ccccc12</smiles>

123

(1) FLPs 27 (5 mol\%)

$\mathrm{H}_{2}$ (4.04 MPa), toluene

$50^{\circ} \mathrm{C}, 24 \mathrm{~h}$

(2) TBAF, toluene, r.t., $1 \mathrm{~h}$

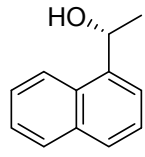

124

yield $=98 \%$ $e e=98 \%$

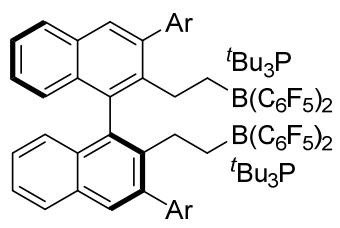

FLPs 26

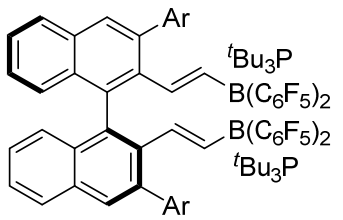

FLPs 27<smiles>CC(C)Cc1cc(C#[Al])cc(C(C)(C)C)c1</smiles>

图 10 手性催化剂 FLPs 26/27

Figure 10 Chiral catalyst FLPs 26/27

原，产物收率为 $52 \% \sim 98 \%, e e$ 值为 $86 \% \sim 99 \%$ (Eq. 41). 值得一提的是, 增加氢源的量, 并没有得到二醇还原副 产物, 表现出较好的化学选择性.
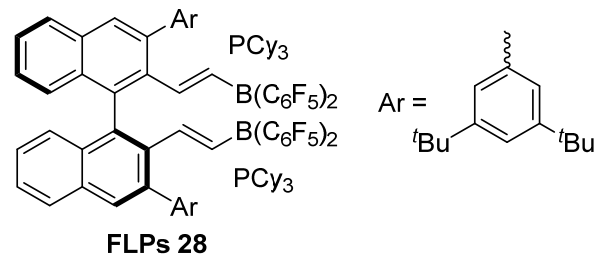

(1) FLPs 28 (5 mol\%)

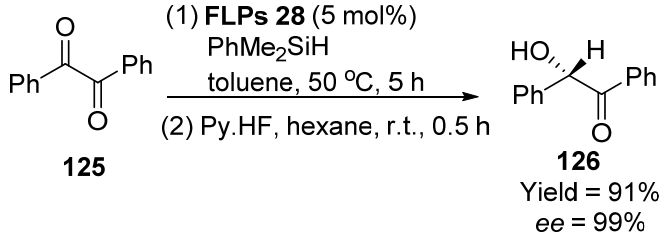

由于 FLPs 催化剂对空间位阻要求较高, 在不对称 还原反应中, 催化剂骨架的选择尤为重要. 目前研究较 多的主要有联菱、樟脑等骨架, 设计具有较大刚性的骨 架对手性 FLPs 催化剂的合成具有重要意义. 基于上述 文献, 我们可以预测: 二茂铁骨架具有平面及中心手 性、足够的结构刚性、合适的立体位阻等诸多优点, 如 果将其作为核心骨架引入 FLPs 催化剂中, 有可能提高 反应的活性和对映选择性, 从而丰富手性 FLPs 催化剂 的类型

\section{6 其它 FLPs 催化氢化反应}

除了上述 FLPs 催化剂之外, 还有其它 FLPs 催化剂 的报道，拓展了 FLPs 的应用范围.

2009 年, Ashley 和 O'Hare 等 ${ }^{[65]}$ 将 FLPs 3 与 $\mathrm{CO}_{2} / \mathrm{H}_{2}$ 混合，在 $160{ }^{\circ} \mathrm{C}$ 下反应 $6 \mathrm{~d}$ 得到甲醇，收率为 $17 \%$ $25 \%$ (Scheme 14). 该反应首次报道了 $\mathrm{CO}_{2}$ 在 FLPs 作用 下转化成甲醇, 尽管收率不高, 但拓展了 FLPs 领域的 新应用, 为 FLPs 在 $\mathrm{CO}_{2}$ 还原反应的研究奠定了基础.

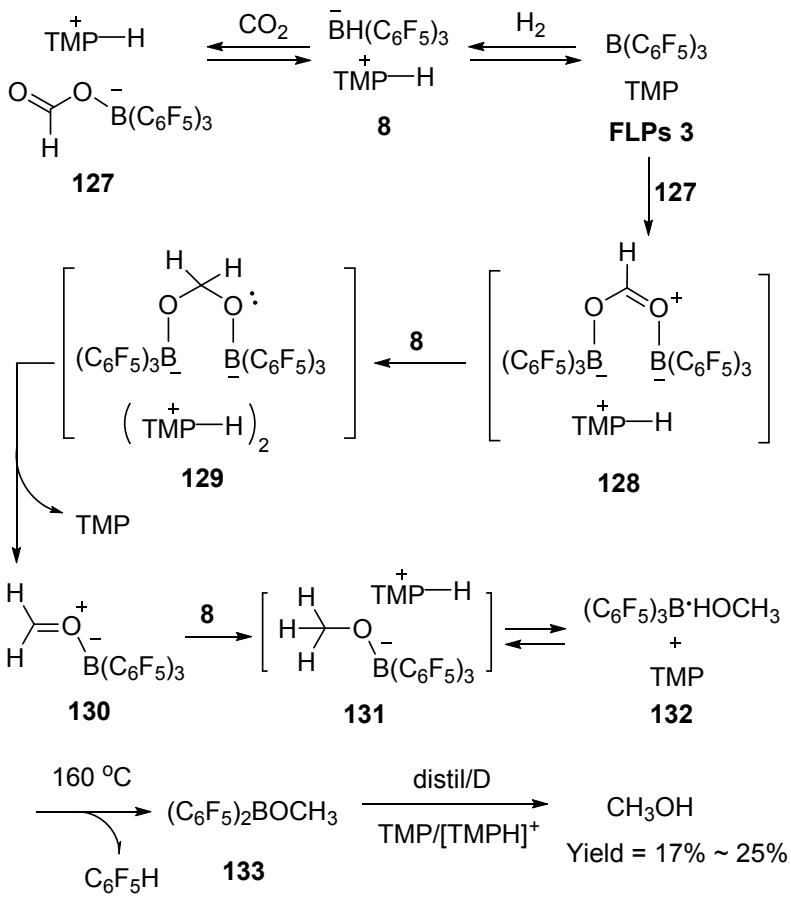

图式 14 FLPs 3 还原 $\mathrm{CO}_{2}$ 成甲醇

Scheme 14 Reduction of $\mathrm{CO}_{2}$ to methanol by FLPs 3

典型的 FLPs 路易斯酸，例如多卤代硼，由于其自 身的缺电子性而具有特殊的反应活性. 2013 年 Stephan 等 ${ }^{[66,67]}$ 报道了电子饱和的 $\mathrm{P}[\mathrm{V}]$ 中心的新型路易斯酸. 他 们以 134 为原料和 $\mathrm{XeF}_{2}$ 反应得到 $\mathrm{P}[\mathrm{V}]$ 135, 再与 $\left[\mathrm{Et}_{3} \mathrm{Si}\right.$ $\left.{ }^{+}\right]$反应脱去一分子氟形成 $\mathrm{P}[\mathrm{V}]$ 离子, 最后与 $\left[\mathrm{B}\left(\mathrm{C}_{6} \mathrm{~F}_{5}\right)_{4}{ }^{-}\right]$ 组成电子饱和的新型 FLPs 29 (Scheme 15). 随后，他们 以 $\mathrm{Et}_{3} \mathrm{SiH}$ 为氢源, 利用该催化剂实现氟代化合物脱氟加 氢反应(Eq. 42). 该反应打破常规思维，创造性地设计了 新型 $\mathrm{P}[\mathrm{V}]$ 路易斯酸，丰富了路易斯酸的种类，为开发新 型 FLPs 路易斯酸提供有益的启示.

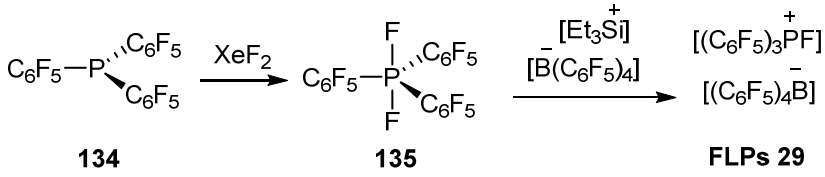

图式 15 FLPs 29 的合成

Scheme 15 Synthesis of FLPs 29 
可能反应机理：首先 FLPs 29 与氟代底物反应，夺 取一分子氟生成 135 , 底物失去一分子氟后与嗍负离子 结合生成两性离子 138, 接着与 $\mathrm{Et}_{3} \mathrm{SiH}$ 反应生成还原产 物 137 和两性离子 139 , 最后 139 夺取物质 135 中的一 分子氟形成 $\mathrm{Et}_{3} \mathrm{SiF}$ 和 FLPs 29 (Scheme 16).
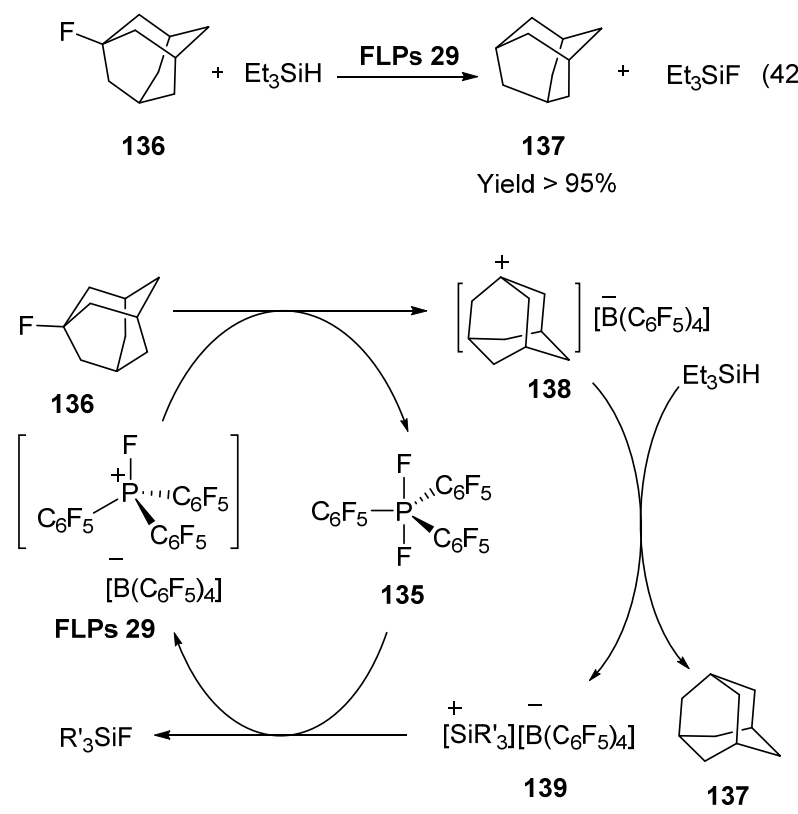

图式 16 FLPs 29 催化脱氟加氢反应的可能机理

Scheme 16 Proposed mechanism of defluorination reaction catalyzed by FLPs 29

2016年, Oestreich等 ${ }^{[68}$ 报道在无需溶剂 $100{ }^{\circ} \mathrm{C}$ 条件 下，利用 1 和 $\mathrm{Et}_{3} \mathrm{SiH}$ 实现硝基化合物的还原，得到 43\% 92\%的收率(Eq. 43). 该反应突破常规, 实现硝基 的还原, 拓展了 FLPs 底物范围, 不足之处是硅氢用量 较大, 且官能团耐受性一般.

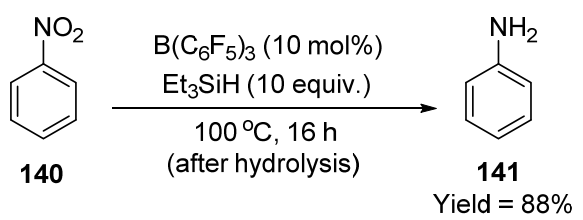

\section{7 总结与展望}

本文主要根据底物类别, 综述了近年来 FLPs 在催 化氢化领域的研究进展, 并对不对称催化氢化领域进行 了介绍. 总的来说, FLPs 催化剂的活性与自身空间位阻 和酸碱性有很大的关系. 适当的空间位阻和酸碱性可以 使催化剂表现出较高活性; 而 P/B 体系 FLPs 相对于 N/B 体系活性更高; 通过调节 Lewis 酸的强弱, 有可能提高 催化剂的官能团耐受性、耐湿性和底物适用性. 在不对 称还原中, 选择具有合适刚性的骨架有可能提高催化剂 活性.
FLPs 催化反应的主要特点是环境友好, 无重金属 残留, 符合绿色化学的要求, 具有潜在的工业化应用前 景. 相比于过渡金属催化, FLP 化学正处于起步阶段, 寻求制备简便、稳定性好、化学选择性高的手性 FLPs 催化剂可能是未来研究的热点. 相信在不久的将来, FLP 会在材料科学、有机催化、不对称合成等诸多领域 发挥独特作用. 因此, 在这个年轻领域的研究过程中, 需要不断突破常规思维, 积极探索, 提出和发展 FLP 领 域的一些新方法和新概念, 为 FLP 领域的科学研究走向 工业应用提供理论基础.

\section{References}

[1] Welch, G. C.; Juan, R. R. S.; Masuda, J. D.; Stephan, D. W. Science 2006, 314, 1124

[2] Spies, P.; Erker, G.; Kehr, G.; Bergander, K.; Fröhlich, R.; Grimme, S.; Stephan, D.W. Chem. Commun. 2007, 5072.

[3] Sumerin, V.; Schulz, F.; Nieger, M.; Leskelä, M.; Repo, T.; Rieger, B. Angew. Chem. Int. Ed. 2008, 47, 6001.

[4] Holschumacher, D.; Bannenberg, T.; Hrib, C. G.; Jones, P. G.; Tamm, M. Angew. Chem. Int. Ed. 2008, 47, 7428.

[5] Lu, Z.-P.; Cheng, Z.-H.; Chen, Z.-X.; Weng, L.-H.; Li, Z.-H.; Wang, H.-D. Angew. Chem., Int. Ed. 2011, 50, 12227.

[6] Zaher, H.; Ashley, A. E.; Irwin, M.; Thompson, A. L.; Gutmann, M. J.; Krämer, T.; O'Hare, D. Chem. Commun., 2013, 49, 9755.

[7] Caputo, C. B.; Zhu, K.-L.; Vukotic, V. N.; Loeb, S. J.; Stephan, D. W. Angew. Chem. Int. Ed. 2013, 52, 960.

[8] Chernichenko, K.; Kótai, B.; Pápai, I.; Zhivonitko, V.; Nieger, M.; Leskelä, M.; Repo; T. Angew. Chem., Int. Ed. 2015, 54, 1749.

[9] Samigullin, K.; Georg, I.; Bolte, M.; Lerner, H. W.; Wagner, M. Chem. Eur. J. 2016, 22, 3478.

[10] Zheng, J.-H.; Lin, Y.-J.; Wang, H.-D. Dalton Trans. 2016, 45, 6088

[11] Mo, Z.-B.; Rit, A.; Campos, J.; Kolychev, E. L.; Aldridge, S. J. Am. Chem. Soc. 2016, 138, 3306

[12] Wang, P.-A.; Sun, X.-L.; Gao, P. Chin. J. Org. Chem. 2011, 31, 1369 (in Chinese).

(王平安，孙晓莉，高鹏，有机化学, 2011, 31, 1369.)

[13] Xu, Y.-Y.; Li, Z.; Maxim B.; Nie, W.-L. Prog. Chem. 2012, 24, 1526 (in Chinese). (徐莹芗，李钊, Maxim B., 聂万丽, 化学进展, 2012, 24, 1526.)

[14] Chase, P. A.; Welch, G. C.; Jurca, T.; Stephan, D. W. Angew. Chem., Int. Ed. 2007, 46, 8050.

[15] Chase, P. A.; Jurca, T.; Stephan, D. W. Chem. Commun. 2008, 1701.

[16] Mohr, J.; Oestreich, M. Angew. Chem., Int. Ed. 2014, 53, 13278.

[17] Wei, S.-M.; Feng, X.-Q.; Du, H.-F. Org. Biomol. Chem. 2016, 14, 8026.

[18] Farrell, J. M.; Heiden, Z. M.; Stephan, D. W. Organometallics 2011, 30, 4497.

[19] Chatterjee, I.; Oestreich, M. Angew. Chem., Int. Ed. 2015, 54, 1965.

[20] Jiang, C.-F.; Blacque, O.; Berke, H. Chem. Commun. 2009, 5518.

[21] Scott, D. J.; Fuchter, M. J.; Ashley, A. E. Angew. Chem., Int. Ed. 2014, 53, 10218.

[22] Spies, P.; Schwendemann, S.; Lange, S.; Kehr, G.; Fröhlich, R.; Erker, G. Angew. Chem., Int. Ed. 2008, 47, 7543.

[23] Wang, G.; Chen, C.; Du, T.-Y.; Zhong, W.-H. Adv. Synth. Catal. 2014, 356, 1747.

[24] (a) Sumerin, V.; Chernichenko, K.; Nieger, M.; Leskelä, M.; Rieger, B.; Repo, T. Adv. Synth. Catal. 2011, 353, 2093.

(b) Chernichenko, K.; Nieger, M.; Leskelä, M.; Repo, T. Dalton 
Trans. 2012, 41, 9029

(c) Sumerin, V.; Schulz, F.; Atsumi, M.; Wang, C.; Nieger, M.; Leskelä, M.; Repo, T.; Pyykkö, P.; Rieger, B. J. Am. Chem. Soc. 2008, 130, 14117.

[25] Farrell, J. M.; Posaratnanathan, R. T.; Stephan, D. W. Chem. Sci. 2015, 6, 2010 .

[26] Mummadi, S.; Unruh, D. K.; Zhao, J.-Y.; Li, S.-H.; Krempner, C. J. Am. Chem. Soc. 2016, 138, 3286.

[27] Schwendemann, S.; Frölich, R.; Kehr, G.; Erker, G. Chem. Sci. 2011, 2, 1842

[28] (a) Parks, D. J.; Spence, R. E. v. H.; Piers, W. E. Angew. Chem., Int. Ed. Engl. 1995, 34, 809

(b) Parks, D. J.; Piers, W. E.; Yap, G. P. A. Organometallics 1998, 17, 5492 .

[29] Wang, H.-D.; Fröhlich, R.; Kehr, G.; Erker, G. Chem. Commun. 2008, 5966.

[30] Greb, L.; Oña-Burgos, P.; Schirmer, B.; Grimme, S.; Stephan, D. W.; Paradies, J. Angew. Chem., Int. Ed. 2012, 51, 10164.

[31] Erõs, G.; Mehdi, H.; Pápai, I.; Rokob, T. A.; Király, P.; Tárkányi, G.; Soós, T. Angew. Chem., Int. Ed. 2010, 49, 6559.

[32] Inés, B.; Palomas, D.; Holle, S.; Steinberg, S.; Nicasio, J. A.; Alcarazo, M. Angew. Chem., Int. Ed. 2012, 51, 12367.

[33] (a) Greb, L.; Daniliuc, C. G.; Bergander, K.; Paradies, J. Angew. Chem., Int. Ed. 2013, 52, 5876.

(b) Paradies, J. Angew. Chem., Int. Ed. 2014, 53, 3552.

[34] Hounjet, L. J.; Bannwarth, C.; Garon, C. N.; Caputo, C. B.; Grimme, S.; Stephan, D. W. Angew. Chem., Int. Ed. 2013, 52, 7492.

[35] Wang, X.-W.; Kehr, G.; Daniliuc, C. G.; Erker, G. J. Am. Chem. Soc. 2014, 136, 3293.

[36] Chernichenko, K.; Madarász, Á.; Pápai, I.; Nieger, M.; Leskelä, M.; Repo, T. Nat. Chem. 2013, 5, 718.

[37] Szeto, K. C.; Sahyoun, W.; Merle, N.; Castelbou, J. L.; Popoff, N.; Lefebvre, F.; Raynaud, J.; Godard, C.; Claver, C.; Delevoye, L.; Gauvinc, R. M.; Taoufik, M. Catal. Sci. Technol. 2016, 6, 882.

[38] Reddy, J. S.; Xu, B.-H.; Mahdi, T.; Fröhlich, R.; Kehr, G.; Stephan, D. W.; Erker, G. Organometallics 2012, 31, 5638.

[39] Greb, L.; Oña-Burgos, P.; Kubas, A.; Falk, F. C.; Breher, F.; Finkc, K.; Paradies, J. Dalton Trans. 2012, 41, 9056.

[40] Longobardi, L. E.; Tang, C.; Stephan, D. W. Dalton Trans. 2014, 43, 15723.

[41] Mahdi, T.; Stephan, D. W. J. Am. Chem. Soc. 2014, 136, 15809.

[42] Scott, D. J.; Fuchter, M. J.; Ashley, A. E. J. Am. Chem. Soc. 2014, 136,15813 .
[43] Mahdi, T.; Stephan, D. W. Angew. Chem., Int. Ed. 2015, 54, 8511.

[44] Gyömöre, Á.; Bakos, M.; Földes, T.; Pápai, I.; Domján, A.; Soós, T. ACS Catal. 2015, 5, 5366.

[45] Geier, S. J.; Chase, P. A.; Stephan, D. W. Chem. Commun. 2010, 46, 4884.

[46] Erös, G.; Nagy, K.; Mehdi, H.; Pápai, I.; Nagy, P.; Király, P.; Tárkányi, G.; Soós, T. Chem. Eur. J. 2012, 18, 574.

[47] Chen, B.-L.; Wang, B.; Lin, G.-Q. J. Org. Chem. 2010, 75, 941.

[48] Segawa, Y.; Stephan, D. W. Chem. Commun. 2012, 48, 11963.

[49] Mahdi, T.; Heiden, Z. M.; Grimme, S.; Stephan, D. W. J. Am. Chem. Soc. 2012, 134, 4088.

[50] Longobardi, L. E.; Mahdi, T.; Stephan, D. W. Dalton Trans. 2015, $44,7114$.

[51] Mahdi, T.; Castillo, J. N. D.; Stephan, D. W. Organometallics 2013, 32, 1971.

[52] Liu, Y.-B.; Du, H.-F. J. Am. Chem. Soc. 2013, 135, 12968.

[53] Liu Y. -B., Du H. -F. Acta Chim. Sinica 2014, 72, 771 (in Chinese). (刘勇兵, 杜海峰, 化学学报, 2014, 72, 771.)

[54] Chen, D.-J.; Klankermayer, J. Chem. Commun. 2008, 2130.

[55] Chen, D.-J.; Wang, Y.-T.; Klankermayer, J. Angew. Chem., Int. Ed. 2010, 49, 9475.

[56] Ghattas, G.; Chen, D.-J.; Pan, F.-F.; Klankermayer, J. Dalton Trans. 2012, $41,9026$.

[57] Chen, D.-J.; Leich, V.; Pan, F.-F.; Klankermayer, J. Chem. Eur. J. 2012, 18, 5184.

[58] Lindqvist, M.; Borre, K.; Axenov, K.; Kótai, B.; Nieger, M.; Leskelä, M.; Pápai, I.; Repo, T. J. Am. Chem. Soc. 2015, 137, 4038.

[59] (a) Mewald, M.; Oestreich, M. Chem. Eur. J. 2012, 18, 14079. (b) Hermeke, J.; Mewald, M.; Oestreich, M. J. Am. Chem. Soc. 2013, 135, 17537.

[60] Süsse, L.; Hermeke, J.; Oestreich, M. J. Am. Chem. Soc. 2016, 138, 6940.

[61] Liu, Y.-B.; Du, H.-F. J. Am. Chem. Soc. 2013, 135, 6810.

[62] Wei, S.-M.; Du, H.-F. J. Am. Chem. Soc. 2014, 136, 12261.

[63] Ren, X.-Y.; Li, G.; Wei, S.-M.; Du, H.-F. Org. Lett. 2015, 17, 990.

[64] Ren, X.-Y.; Du, H.-F. J. Am. Chem. Soc. 2016, 138, 810.

[65] Ashley, A. E.; Thompson, A. L.; O'Hare, D. Angew. Chem., Int. Ed. 2009, 48, 9839.

[66] Caputo, C. B.; Hounjet, L. J.; Dobrovetsky, R.; Stephan, D. W. Science 2013, 341, 1374.

[67] Hounjet, L. J.; Caputo, C. B.; Stephan, D. W. Dalton Trans. 2013, 42, 2629.

[68] Porwal, D.; Oestreich, M. Eur. J. Org. Chem. 2016, 3307.

(Li, L.) 